\title{
Clustering-mediated activation of Cdc42 GTPase antagonized by GAPs in fission yeast
}

Iker Lamas, Nathalie Weber and Sophie G Martin*

Department of Fundamental Microbiology, Faculty of Biology and Medicine, University of Lausanne, Biophore building, 1015 Lausanne, Switzerland

* author for correspondence: Sophie.Martin@unil.ch

\begin{abstract}
The small GTPase Cdc42 is critical for cell polarization in eukaryotic cells. In rod-shaped fission yeast Schizosaccharomyces pombe cells, active GTP-bound Cdc42 promotes polarized growth at cell poles, while inactive Cdc42-GDP localizes ubiquitously also along cell sides. Zones of Cdc42 activity are maintained by positive feedback amplification involving the formation of a complex between Cdc42GTP, the scaffold Scd2 and the guanine nucleotide exchange factor (GEF) Scd1, which promotes the activation of more Cdc42. Here, we use the CRY2-CIB1 optogenetic system to recruit and cluster a cytosolic Cdc42 allele at the plasma membrane and show that this leads to its moderate activation also on cell sides. Surprisingly, activation of CRY2-Cdc42 does not individually depend on Scd1 or the GEF Gef1. We show that activated Cdc42 clusters at cell sides are able to recruit Scd1, dependent on the scaffold Scd2. However, Cdc42 activity is not amplified by positive feedback and does not lead to morphogenetic changes, due to antagonistic activity of the GTPase activating protein Rga4 on cell sides. Thus, the cell architecture is robust to moderate activation of Cdc42 at cell sides.
\end{abstract}




\section{Introduction}

In eukaryotes, the small Rho-family GTPase Cdc42 is a highly conserved regulator of cell morphogenesis, proliferation and differentiation. Prenylation of Cdc42's C-terminal CAAX motif underlies its association with the plasma membrane, where it functions as a molecular switch that alternates between GTP-bound, active and GDP-bound, inactive states. Activation of Rho GTPases relies on the activity of guanine nucleotide exchange factors (GEFs), while their intrinsic GTPase activity is enhanced by GTPase activating proteins (GAPs) to return them to the inactive state. GDP-bound Cdc42 also binds guanine-nucleotide dissociation inhibitors (GDI), which both block the exchange of GDP by GTP and solubilize Cdc42-GDP in the cytosol.

In the fission yeast Schizosaccharomyces pombe, Cdc42 is active at sites of polarized growth during vegetative and sexual life cycles. GTP-loading is promoted by two GEFs, Scd1 and Gef1. Scd1, which localizes to cell poles, receives information from the upstream Ras1 GTPase signal and mediates feedback control through the scaffolding activity of Scd2 (Bendezu et al., 2015; Kelly and Nurse, 2011; Lamas et al., 2020). For this, Scd1 forms a quaternary complex with Cdc42-GTP, the Pak1 kinase effector and Scd2 (Chang et al., 1994; Endo et al., 2003), which leads in vivo to the positive feedback activation of other Cdc42 molecules, as shown in our recent work using optogenetic strategies (Lamas et al., 2020). The second GEF, Gef1, which localizes to cell poles only in some conditions, promotes Cdc42 activation in response to stress and becomes essential only in absence of Scd1 (Coll et al., 2003; Hirota et al., 2003; Tay et al., 2018; Vjestica et al., 2013). Three GTPase activating proteins (GAPs) Rga4, Rga6 and Rga3 enhance the intrinsic GTP hydrolytic activity of Cdc42 (Das et al., 2007; Gallo Castro and Martin, 2018; Revilla-Guarinos et al., 2016). Rga4 and Rga6 GAPs localize at cell sides, where growth does not occur in non-stressed cells, whereas Rga3 localizes at sites of active growth (cell poles). Fission yeast cells also express a GDI, called Rdi1, though Cdc42 localization and dynamics are not strongly perturbed in its absence (Bendezu et al., 2015; Nakano et al., 2003).

Recently, optogenetic studies revealed a novel mechanism that triggers the activation of small GTPases in mammalian cells: Human Rac1 and RhoA, which belong to the same Rho GTPase family as Cdc42, were shown to become active at the cell cortex upon light-dependent cytosolic clustering (Bugaj et al., 2013). In these experiments, the small GTPases were fused to CRY2PHR, the photolyase homology region of $A$. thaliana cryptochrome 2 , which oligomerizes upon blue light exposure. Artificially clustered RhoA induced RhoA signalling-dependent cytoskeletal re-organization and membrane retraction in human cells, suggesting that oligomerization promotes RhoA activation (Bugaj et al., 2013). Ras and Ras-like GTPases are well known to form nanoclusters and dimers at the membrane to activate signal transduction (Inouye et al., 2000; Kang et al., 2010; Nussinov et al., 2020). Several Rho-family GTPases, including RhoA, Rac1, Rac2 and Cdc42, were also shown to form dimers or oligomers through homophilic interactions of their polybasic region adjacent to the C-terminal CAAX motif (Zhang et al., 2001; Zhang and Zheng, 1998). While oligomerization of GTP-bound Cdc42 and Rac1 increases their GTPase activity in vitro, the physiological relevance of clustering of these small GTPases remains to be investigated (Zhang and Zheng, 1998). In vivo, Rac1-GTP oligomers have been shown to contain several dozen Rac1 molecules together with charged phospholipids and appear to promote signal 
transduction (Maxwell et al., 2018; Remorino et al., 2017; Zhang et al., 2001). Cdc42 also forms nanoclusters in Saccharomyces cerevisiae cells (Slaughter et al. 2013; Sartorel et al. 2018). These nanoclusters show an anisotropic distribution: they accumulate and exhibit larger sizes at cortical sites of polarized growth, in a manner dependent on the scaffold protein Bem1 and anionic membrane lipids (Meca et al., 2019; Sartorel et al., 2018). Because Bem1 also acts as scaffold that bridges Cdc42-GTP to its GEF, thereby promoting positive feedback activation of $\mathrm{Cdc} 42$, Cdc42 nanoclusters may promote feedback Cdc42 activation, though this has not been tested.

In this work, we used an artificial optogenetic strategy to induce the recruitment and clustering of Cdc42 at the plasma membrane of fission yeast cells. We built on our recent work that used the CRY2-CIB1 optogenetic system to probe the positive feedback of Cdc42 (Lamas et al., 2020). The CRY2-CIB1 system relies on the blue light-induced binding of CRY2PHR (simply denoted CRY2 below) to the Nterminal part of CRY2-binding partner CIB1 (CIBN) (Kennedy et al., 2010). Blue light also induces the formation of CRY2 oligomers (Bugaj et al., 2013). We fused CRY2 to a cytosolic allele of Cdc42 $\left(\mathrm{Cdc} 42^{\triangle \mathrm{Caax}}\right)$ and co-expressed CIBN linked to the membrane-associated RitC anchor. Cortical recruitment of a GTP-locked, constitutively active Cdc42 allele (CRY2-Cdc42 ${ }^{\mathrm{Q61L}, \Delta \mathrm{CaaX}}$ ) led to the Scd2dependent co-recruitment of its GEF Scd1 and accumulation of endogenous Cdc42, demonstrating feedback amplification. Surprisingly, cortical recruitment of CRY2-Cdc42 ${ }^{\triangle \mathrm{CaaX}}$ (not GTP-locked) also induced the co-recruitment of Scd2 (Lamas et al, 2020), suggesting activation of CRY2-Cdc42 ${ }^{\mathrm{ACaax}}$. Here, we confirm that CRY2-dependent clustering promotes Cdc42 activation at lateral sites, where Cdc42 is normally inactive. Activated clustered Cdc42 is able to recruit its GEF through the scaffold Scd2, suggesting that positive feedback is initiated. However, the activation is efficiently countered by Rga4 GAP-mediated Cdc42 inactivation, and does not lead to cell shape alteration, showing the robustness of the cell polarization system.

\section{Results and discussion}

\section{Weak activation of CRY2-Cdc42 at the cell cortex}

To better characterize CRY2-Cdc42 ${ }^{\triangle \mathrm{CaaX}}$, we first measured its kinetics of recruitment to CIBN-RitC at the plasma membrane. Similar to rates measured for CRY2, CRY2-Cdc42 ${ }^{\triangle \mathrm{CaaX}}$ showed a half-time of protein recruitment to the cortex $<1 \mathrm{~s}$ and independent of the length of the blue light $(488 \mathrm{~nm})$ pulses (30 GFP pulses of $50 \mathrm{~ms}=0.92 \mathrm{~s} \pm 0.24 \mathrm{~s} ; 22$ GFP pulses of $250 \mathrm{~ms}=0.98 \mathrm{~s} \pm 0.25 \mathrm{~s} ; 17$ GFP pulses of $500 \mathrm{~ms}=0.99 \mathrm{~s} \pm 0.31 \mathrm{~s}$; Fig S1A-B). CRY2-Cdc42 ${ }^{\Delta \mathrm{CaaX}}$ cells did not exhibit any morphological defects and grew in a bipolar fashion in the dark (Fig S1C). In blue-light, CRY2-Cdc42 ${ }^{\triangle \mathrm{CaaX}}$ cells maintained their characteristic rod-shaped and continued growing from the cell tips (Fig S1D - green cells), while cells with activated CRY2-Cdc42 ${ }^{\mathrm{Q} 61 \mathrm{~L}, \Delta \mathrm{CaaX}}$ rounded up indicating isotropic growth (Fig S1D - blue cells; (Lamas et al., 2020)). These evidences initially suggested that the recruitment of CRY2Cdc42 ${ }^{\Delta \mathrm{CaaX}}$ to the cell cortex was innocuous and unable to bias the endogenous Cdc42 and its regulatory network. 
We monitored the distribution of Cdc42-GTP using three GFP-tagged markers that specifically associate with Cdc42-GTP: the scaffold Scd2 (Endo et al., 2003; Wheatley and Rittinger, 2005), the CRIB bioreporter (Tatebe et al., 2008), and the Cdc42 effector Pak1, which also contains a CRIB domain. These proteins and probe are normally only detected at the poles and division sites of yeast cells, as well as weakly in the nucleus for the first two. As previously described (Lamas et al 2020), upon blue light-dependent recruitment of CRY2-Cdc42 ${ }^{\triangle \mathrm{Caax}}$ to the plasma membrane, Scd2-GFP formed stable foci at the cell sides, which increased progressively in intensity, while Scd2-GFP intensity decreased at cell poles (Fig 1A-B). CRIB-3GFP also formed dim, dynamic foci at the cell sides, which became visible 40-60s after light stimulation (Fig 1A-B). The CRIB-3GFP side signal was however weaker and delayed relative to that observed upon light-induced recruitment of GTP-locked CRY2Cdc42 ${ }^{\mathrm{Q} 61 L, \Delta \mathrm{CaaX}}$ (Fig 1C; (Lamas et al., 2020)). The Pak1-sfGFP trace on cell sides also showed an upward trajectory, but was not statistically different from negative control after 90 s, suggesting delayed recruitment below the detection threshold (Fig 1A-C). Together, these data suggest that the heterologous allele of $\mathrm{Cdc} 42$ within the CRY2-Cdc42 ${ }^{\Delta \mathrm{Caax}}$ system is transiently activated when recruited in clusters at the cell sides.

As an alternative strategy to increase Cdc42 levels at the plasma membrane, we overexpressed Cdc42. In this experiment, we used the functional, internally tagged Cdc42-mCherry ${ }^{s w}$ allele (Bendezu et al., 2015) expressed under the $p^{\text {act } 1}$ promoter in cells lacking the endogenous gene, which allowed us to quantify the global increase in expression levels at 3.3-fold (Fig S2A-B). The Cdc42 level increase was roughly uniform around the cell cortex (not shown). Cdc42 overexpression also led to a 1.2-fold increase in expression of the CRIB-3GFP reporter (expressed under the $p^{\text {pak1 }}$ promoter, Fig S2B). Cdc42 overexpression led to a small increase in CRIB signal at cell poles (even after correction by the 1.2-fold increase in probe expression) and a small increase in cell length ( $p^{\text {act1 }}$-cdc42 cell length $=14.2 \pm 1 \mu \mathrm{m}$ vs WT cell length $=13.4 \pm 1.1 \mu \mathrm{m}$, t-test $p$-value $=2.8 \mathrm{e}-5 ; p^{\text {act } 1}$-cdc42 cell width $=3.8 \pm 0.3 \mu \mathrm{m}$ vs WT cell width $=3.8 \pm 0.3 \mu \mathrm{m}$, t-test $\mathrm{p}$-value $=0.96$ ), suggesting increased Cdc42 activity at cell poles. However, Cdc42 overexpression had no effect on CRIB-3GFP levels at cell sides (Fig S2C-D). We conclude that activation of $\mathrm{CRY} 2-\mathrm{Cdc} 42^{\Delta \mathrm{CaaX}}$ on cell sides is not simply a consequence of overexpression but may be due to forced CRY2-dependent clustering of Cdc42.

\section{CRY2-Cdc42 activation still occurs in absence of either Cdc42 GEF}

To probe the mode of CRY2-Cdc42 ${ }^{\triangle \mathrm{CaaX}}$ activation, we repeated the optogenetic experiments above in strains lacking the Cdc42 GEF Scd1. In scd1 $\Delta$ cells, Pak1-sfGFP was not detected at cell sides, as expected. We also observed only rare CRIB-3GFP dots, and no significant increase in CRIB levels at the sides of $s c d 1 \Delta$ cells (Fig $2 A-B$ ), suggesting Scd1 may participate in CRY2-Cdc42 ${ }^{\Delta C a a X}$ activation. However, Scd2-GFP was still recruited to cell sides (Fig 2A-B), indicating Scd1 is not the sole activator of Cdc42 in these cells. We thus probed the role of the second Cdc42 GEF Gef1. In gef1 $\Delta$ cells, Scd2GFP accumulation at cell sides was not affected and exhibited similar re-localization dynamics as in WT cells (Fig 2C-D). We conclude that neither Cdc42 GEF is individually required for initial activation of clustered Cdc42 at the plasma membrane. It is possible that the two GEFs work redundantly in this 
situation. The lethality of $s c d 1 \Delta$ gef1 $1 \Delta$ double mutants unfortunately does not allow testing of this hypothesis (Coll et al., 2003; Hirota et al., 2003). Alternatively, Cdc42 clustering through CRY2 binding may promote its activation in GEF-independent manner.

\section{CRY2-Cdc42 promotes recruitment of its GEF Scd1 in Scd2 scaffold-dependent manner}

Because Cdc42-GTP promotes the recruitment of its GEF Scd1 for feedback amplification of Cdc42 activation (Lamas et al 2020), we probed whether CRY2-Cdc42 ${ }^{\Delta \mathrm{CaaX}}$ induces Scd1 recruitment. Indeed, Scd1 formed weak, dynamic foci at cell sides upon blue-light activation, similar to the CRIB-3GFP foci described above (Fig 3A-B). The appearance of Scd1 dynamic foci at cell sides was also mirrored by a decrease of Scd1-3GFP at the cell tips (Fig 3C). Scd1 recruitment was dependent on the scaffold Scd2, as no cell side accumulation of Scd1-3GFP, nor decrease at cell tips, was detected in scd2 $\Delta$ cells (Fig $3 \mathrm{D}-\mathrm{F})$. These data suggest that the activated CRY2-Cdc42 $4 \mathrm{CaaX}$ is poised to trigger the positive feedback leading to recruitment of its GEF Scd1.

Although scd2 deletion abolished Scd1 recruitment, it did not substantially affect the accumulation of the CRIB probe (Fig 3D, 3G-H), indicating that CRY2-Cdc42 ${ }^{\triangle \mathrm{CaaX}}$ is still activated in these cells. This observation is in agreement with the finding that CRY2-Cdc42 ${ }^{\triangle \mathrm{CaaX}}$ is activated independently of Scd1. Because CRIB intensity on cell sides was not reduced in scd2 $\Delta$ cells (Fig $3 \mathrm{H}$ ), we conclude that the scaffold-dependent recruitment of the GEF by CRY2-Cdc42 ${ }^{\triangle \mathrm{CaaX}}$ does not play a major role in amplifying Cdc42 activation at cell sides.

\section{The Cdc42 GAP Rga4 prevents isotropic growth of CRY2-Cdc42 ${ }^{\triangle \mathrm{CaaX}}$ cells}

If CRY2-Cdc42 $2^{\Delta \mathrm{CaaX}}$ is activated at cell sides and recruits its own GEF, why is Cdc42 activity not further amplified by the positive feedback mechanism and does not lead to cell shape changes? Indeed, even long-term growth of CRY2-Cdc42 cells in the light did not change their cell length and width, or aspect ratio, similar to control CRY2 cells. By contrast, constitutive cortical localization of CRY2-Cdc42 ${ }^{\mathrm{Q} 61 \mathrm{~L}, \mathrm{ACaaX}}$ by growth in light conditions led to significant increase in cell width and decrease in cell length, yielding a reduced aspect ratio (Fig $4 \mathrm{~A}-\mathrm{C}$ ).

We hypothesized that the activation of $\mathrm{CRY} 2-\mathrm{Cdc} 42^{\triangle \mathrm{CaaX}}$ at cell sides is rapidly counteracted by negative regulators. We focused our attention on the three Cdc42 GAPs Rga3, Rga4 and Rga6, the GDI protein Rdi1 and the Ras1 GAP Gap1. Rga3, Rga4 and Rga6 directly promote Cdc42-GTP hydrolysis (Das et al., 2007; Gallo Castro and Martin, 2018; Revilla-Guarinos et al., 2016). Rdi1 may promote Cdc42 extraction from the membrane, although it is largely dispensable for Cdc42 dynamics (Bendezu et al., 2015; Nakano et al., 2003). Gap1 directly promotes Ras1-GTP hydrolysis (Merlini et al., 2018). As Ras1 promotes Scd1 activation and is uniformly active at the plasma membrane in gap $1 \Delta$ (Lamas et al., 2020; Merlini et al., 2016; Merlini et al., 2018), we hypothesized Scd1 activation on cell sides may be amplified in this mutant. We constructed single and most double deletion mutants expressing either of $\mathrm{CRY} 2-\mathrm{Cdc} 42^{\Delta \mathrm{CaaX}}, \mathrm{CRY} 2-\mathrm{Cdc} 42^{\mathrm{Q} 61 \mathrm{~L}, \Delta \mathrm{CaaX}}$, as positive control or CRY2 alone as 
negative control. We then measured the cell length and cell width of calcofluor-stained dividing cells after at least $14 \mathrm{~h}$ of exponential growth in light conditions and calculated aspect ratios (Fig 4A).

To estimate the change in aspect ratio upon Cdc42 lateral recruitment while taking into account the initial shape of the cell, we normalized the aspect ratios from cells recruiting Cdc42 to those expressing only CRY2 (Fig 4B). CRY2-Cdc42 ${ }^{\mathrm{Q} 61 \mathrm{~L}, \Delta \mathrm{CaaX}}$ led to a $>2$-fold reduction in aspect ratio in WT, rga6 $\Delta, r d i 1 \Delta$ and gap $1 \Delta$ cells, but had less effect on cell shape change in single and double rga $4 \Delta$ mutants, perhaps in part due to the already wider cell shape of rga4 4 cells (Das et al., 2007). Interestingly, CRY2Cdc42 ${ }^{\Delta \mathrm{CaaX}}$ had little effect on aspect ratio in WT or any single mutants, except in rga4 $\Delta$ cells, which became significantly rounder. Similar, more marked effects were also observed in combinations of rga4 $\Delta$ with rga6 $\Delta$ or rdi1 $\Delta$. The effect of CRY2-Cdc42 ${ }^{\Delta \mathrm{CaaX}}$ on the shape of these mutants can also readily be observed in plots of cell width to cell length, with the CRY2-Cdc42 ${ }^{\triangle \mathrm{CaaX}}$ cell population placed at an intermediate position between the negative CRY2 and positive CRY2-Cdc42 ${ }^{\mathrm{Q} 61 \mathrm{~L}, \Delta \mathrm{CaaX}}$ controls (Fig 4C). These data indicate that the optogenetic-dependent Cdc42 activation is counteracted by Rga4 GAP placed at cell sides.

In summary, the data presented in this work show that CRY2-clustered Cdc42 is ectopically activated at cell sides. It is possible that CRY2-tagging interferes with Cdc42 GTPase activity, such that CRY2Cdc42 ${ }^{\triangle \mathrm{CaaX}}$ represents a slightly activated Cdc42 allele. Alternatively, the CRY2-dependent clustering may lead to Cdc42 activation. This mirrors similar observations made with other GTPases, such as Rac1 and RhoA in mammalian cells (Bugaj et al., 2013). CRY2-mediated Cdc42 activation is not dependent on either individual Cdc42 GEF, though it is possible that both play a redundant role. The activity level of CRY2-Cdc42 at cell sides is sufficient to recruit the GEF Scd1, whose recruitment to Cdc42-GTP depends on the scaffold Scd2 (Lamas et al., 2020), but positive feedback does not appear to become established as $\mathrm{Cdc42}$ activity levels at cell sides are not altered in absence of Scd2. It is possible that the non-physiological linkage of $\mathrm{Cdc} 42$ to the plasma membrane (through RitC-CIBNCRY2 binding instead of through the normal prenyl group) undermines the positive feedback, although we previously showed that a similarly engineered constitutively active $\mathrm{Cdc} 42$ allele did trigger feedbackdependent recruitment of endogenous Cdc42 (Lamas et al., 2020). Alternatively, cellular regions may not be equally permissive to Cdc42 activity feedback amplification. For instance, the absence of Ras 1 activity on cell sides may reduce the effectiveness of the feedback (Lamas et al., 2020). Conversely, we have shown here that the Cdc42 GAP Rga4, which is enriched on cell sides, antagonizes the effect of CRY2-Cdc42 on cell morphogenesis. Additional mechanisms preventing growth on cell sides are also being proposed (Miller et al, BioRxiv). Thus, one critical question for future research is to better understand the many layers that confer robustness to cell morphogenesis.

\section{Materials and methods}

\section{Strains, Media, and Growth Conditions}

Strains used in this study are listed in Table S1. Standard genetic manipulation methods for S. pombe transformation and tetrad dissection were used to generate the strains listed. For microscopy 
experiments, cells were first pre-cultured in $3 \mathrm{~mL}$ of Edinburgh minimal media (EMM) in dark conditions at $30^{\circ} \mathrm{C}$ for $6-8 \mathrm{~h}$. Once exponentially growing, pre-cultures were diluted (Optical Density (O.D.) 600nm $=0.02$ ) in $10 \mathrm{~mL}$ of EMM and incubated in dark conditions overnight at $30^{\circ} \mathrm{C}$. In order to allow proper aeration of the culture, $50 \mathrm{~mL}$ Erlenmeyer flasks were used. For cell size analyses cells were precultured and diluted once in $3 \mathrm{~mL}$ of Edinburgh minimal media (EMM) in dark conditions at $30^{\circ} \mathrm{C}$ for 6 $-8 \mathrm{~h}$. Once exponentially growing, pre-cultures were diluted in $10 \mathrm{~mL}$ of EMM and incubated in light conditions for a minimum of $14 \mathrm{~h}$. All live-cell imaging was performed on EMM-ALU agarose pads, except calcofluor-white experiments in which cells were placed directly on a slide (Dudin et al., 2015). Gene tagging was performed at endogenous genomic locus at the 3' end, yielding C-terminally tagged proteins, as described (Bähler et al., 1998). Pak1 gene tagging was performed by transforming a WT

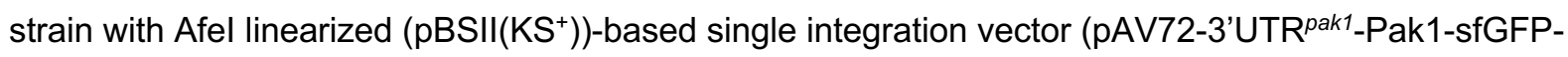
kanMX-5'UTR ${ }^{\text {pak1}}$ ) targeting the endogenous locus. The functional mCherry-tagged and sfGFP-tagged Cdc42 alleles Cdc42-mCherry ${ }^{\text {sw }}$ and Cdc42-sfGFPsw were used as described in (Bendezu et al., 2015). Gene tagging, deletion and plasmid integration were confirmed by diagnostic PCR for both sides of the gene.

Construction of plasmids and strains expressing CIBN-mTagBFP2-Ritc, CRY2, CRY2-Cdc42 ${ }^{\triangle \mathrm{CaaX}}$ and CRY2-Cdc42 ${ }^{\mathrm{Q} 61 \mathrm{~L}, \Delta \mathrm{CaaX}}$ was done as described in (Lamas et al. 2020).

To generate the Pact1-cdc42-mCh ${ }^{S W}$ strain a pINT-ura4+ integrative vector was generated. Pact1 was amplified from gDNA using primers osm2378 (atgggcccgctagcatgcGATCTACGATAATGAGACGG) and osm2379 (ccggctcgagGGTCTTGTCTTTTGAGGGTT) and cloned using Apal and Xhol. Cdc42-mCh ${ }^{\text {sw }}$

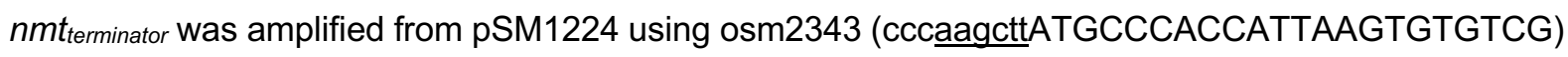
and osm2344 (gctctagaCTTCTAATTACACAAATTCCG) and cloned using Hindlll and Xbal. As a results pSM1449 was generated. This plasmid was linearized with Avrll and integrated at ura4 locus of YSM485 strain. The endogenous allele of $c d c 42$ was deleted using a hygromycin $\left(h p h^{+}\right)$resistance cassette as described (Bähler et al., 1998). $\mathrm{Hph}^{+}$cassette was amplified from pSM693 using osm2511 (TACTTAGGGGTTTGAACTTTCTAGGAATTCAATAAAGTGAAGCAAAGCTTTACGATTAATTATTTT TTGTGAAATAGTcggatccccgggttaattaa) and osm2512 (AAGCTAAGACATTGTTTACTGTTGTAAACTAGCTGTATTAAGGAAATTTCGGAAAAGGAAAGAAA ACCAGGGGTTAAAgaattcgagctcgtttaaac). Finally, strain YSM3732 was generated by transforming the Cdc42-mCh ${ }^{S W_{-}} n \mathrm{tt}_{\text {terminator }}$ strain with the $h p h^{+}$resistance cassette.

In primer sequences, restriction sites are underlined. Plasmid maps are available upon request.

\section{Cell length and width measurements}

For cell length and width measurements shown in Fig 4 and Fig S1, cells were grown at $30^{\circ} \mathrm{C}$ in $10 \mathrm{ml}$ EMM in light and dark conditions respectively. Exponentially growing cells were stained with calcofluor to visualize the cell wall and imaged on a Leica epifluorescence microscope with 60x magnification 
platform described previously (Dudin et al., 2015). Measurements were made with ImageJ on septating cells. For each experiment strains with identical auxotrophies were used.

Aspect ratios were calculated as:

$$
\text { Aspect ratio }=\text { Cell length } / \text { Cell width }
$$

The index of ratios shown in Fig 4B was calculated as:

$$
\begin{gathered}
\text { Index }_{\text {Cdc42 } \triangle \text { CaaX }}=\operatorname{Aspect} \text { ratio }(C R Y 2 C d c 42 \Delta C a a X) / \text { Aspect ratio }(C R Y 2) \\
\text { Index }_{\text {Cdc42Q61L } \triangle \text { CaaX }}=\text { Aspect ratio }(C R Y 2 C d c 42 \Delta Q 61 L C a a X) / \text { Aspect ratio }(C R Y 2)
\end{gathered}
$$

\section{Microscopy}

Fluorescence microscopy experiments were done in a spinning disk confocal microscope, essentially as described (Bendezu and Martin, 2011; Dudin et al., 2015). Image acquisition was performed on a Leica DMI6000SD inverted microscope equipped with an HCX PL APO 100X/1.46 numerical aperture oil objective and a PerkinEImer Confocal system. This system uses a Yokagawa CSU22 real-time confocal scanning head, solid-state laser lines and a cooled 14-bit frame transfer EMCCD C9100-50 camera (Hamamatsu) and is run by Volocity (PerkinElmer). When imaging strains expressing the CRY2-Cdc42 ${ }^{\triangle \mathrm{CaaX}}$ and/or CRY2 systems, an additional long-pass color filter (550 nm, Thorlabs Inc, USA) was used for bright-field (BF) image acquisition, in order to avoid photo-activation by white light.

Spinning disk confocal microscopy experiments shown in Fig 1,2 and 3 were carried out using cell mixtures (Lamas et al. 2020). Cell mixtures were composed by one strain of interest (the sample optogenetic strain, expressing or not an additional GFP-tagged protein) and 2 control strains, namely:

1) RFP control: An RFP bleaching correction strain, expressing cytosolic CRY2PHR-mCherry.

2) GFP control: A wild type strain expressing the same GFP-tagged protein as the strain of interest but without the optogenetic system. This strain was used both as negative control for cell side re-localization experiments and as GFP bleaching correction strain (in Fig 1-2-3 and related supplementary figures).

Strains were handled in dark conditions throughout. Red LED light was used in the room in order to manipulate strains and to prepare the agarose pads. Strains were cultured separately. Exponentially growing cells (O.D.600nm $=0.4-0.6$ ) were mixed with 2:1:1 (strain of interest, RFP control and GFP control) ratio, and harvested by soft centrifugation (2 min at 1,600 rpm). $1 \mu \mathrm{L}$ of the cell mixture slurry was placed on a $2 \%$ EMM-ALU agarose pad, covered with a \#1.5-thick coverslip and sealed with VALAP (vaseline, lanolin and paraffin). Samples were imaged after $5-10$ minutes of rest in dark conditions. 
The plasma membrane recruitment dynamics of CRY2-Cdc42 ${ }^{\Delta \mathrm{CaaX}}$ and CRY2 systems were assessed using cell mixtures. Protein recruitment dynamics were assessed by applying the 3 different photoactivating cycles listed below. Lasers were set to $100 \%$; shutters were set to maximum speed and in all instances the RFP channel was imaged first, before the GFP channel. The duration of the experiment was equal regardless of the exposure time settings $(\approx 15 \mathrm{~s})$ :

- $\quad 50$ ms: RFP channel (200 ms), GFP channel ( $50 \mathrm{~ms})$. This constitutes one cycle $(\approx 0.5 \mathrm{~s}) .30$ time points were acquired $(\approx 0.5 \mathrm{~s} * 30=15.1 \mathrm{~s})$.

- $\quad 250$ ms: RFP channel $(200 \mathrm{~ms})$, GFP channel $(250 \mathrm{~ms})$. This constitutes one cycle $(\approx 0.7 \mathrm{~s}) .22$ time points were acquired $(\approx 0.7 \mathrm{~s} * 22=15.1 \mathrm{~s})$.

- $\quad 500$ ms: RFP channel (200 ms), GFP channel (500 ms). This constitutes one cycle $(\approx 0.9 \mathrm{~s}) .17$ time points were acquired $(0.9 \mathrm{~s} * 17=15.5 \mathrm{~s})$.

Endogenous GFP-tagged protein re-localization experiments were carried out using cell mixtures. Lasers were set to $100 \%$; shutters were set to sample protection and in all instances the RFP channel was imaged first and then the GFP channel. RFP exposure time was always set to $200 \mathrm{~ms}$, whereas the GFP exposure time varied depending on the monitored protein. Cells were monitored in these conditions for $90 \mathrm{~s}$.

Spinning disk confocal time (sum) projections of five consecutive single plane images are shown in Fig S2. Z-stack images were acquired on a Spinning disk confocal microscope using an optimal z-spacing of $0.71 \mu \mathrm{m}$ between successive stacks. 6 stacks were acquired (covered $Z$ distance $=4 \mu \mathrm{m}$ ).

\section{Image Analysis}

All image-processing analyses were performed with Image $\mathrm{J}$ software (http://rsb.info.nih.gov/ij/). Image and time-lapse recordings were imported to the software using the Bio-Formats plugin (http://loci.wisc.edu/software/bio-formats). Time-lapse recordings were aligned using the StackReg plugin (http://bigwww.epfl.ch/thevenaz/stackreg/) according to the rigid body method. All optogenetic data analyses were performed using MATLAB (R2019a), with scripts developed in-house.

\section{CRY2-Cdc42 ${ }^{\triangle \mathrm{CaaX}}$ and CRY2 quantifications:}

The plasma membrane recruitment dynamics of CRY2-Cdc42 ${ }^{\triangle \mathrm{CaaX}}$ and CRY2_systems was assessed by recording the fluorescence intensity over a 15 pixel long by 36 pixel wide ROI (roughly $1.25 \mu \mathrm{m}$ by $3 \mu \mathrm{m}$ ), drawn perpendicular to the plasma membrane of sample cells, from outside of the cell towards the cytosol. The fluorescence intensity values across the length of the ROI were recorded over time in the RFP channel, in which each pixel represents the average of the width ( 36 pixels) of the ROI ( 3 replicates, 30 cells per replicate). Average background signal was measured from tag-free wild-type 
cells incorporated into the cell mixture. The total fluorescence of the Control RFP strain was also measured over time in order to correct for mCherry fluorophore bleaching. In both cases, the ROI encompassed whole cells, where ROI boundaries coincide with the plasma membrane.

Photobleaching correction coefficient was calculated by the following formula:

(1) RFP bleaching correction coefficient $=\left(R F P\right.$ Intensity $\left._{t n}-N o G F P B c k g_{t n}\right) /\left(R F P\right.$ Intensity $\left.y_{t 0}-N o G F P B c k g_{t 0}\right)$

where RFP intensity is the signal measured from single RFP control cells, NoGFPBckg is the average background signal measured from tag-free cells, $t_{n}$ represents a given time point along the time course of the experiment and to represents the initial time point ( $n=30$ time points). These coefficients were corrected by a moving average smoothing method (moving averaged values $=5$ ). RFP bleaching correction coefficient values calculated for individual RFP control cells were averaged in order to correct for bleaching of the RFP signal.

The fluorescence intensity values of optogenetic cells were corrected at each time point with the following formula:

(2) RFP intensity $=\left(\left(\right.\right.$ Raw RFP signal $\left.{ }_{t n}-N_{o G F P B c k g_{t n}}\right) /$ RFP bleaching correction coef ficient $\left._{\text {tn }}\right)$

where Raw RFP signal is for the RFP values measured from sample strains, NoGFPBckg is the average background signal measured from tag-free cells and $t_{n}$ represents a given time point along the time course of the experiment ( $n=30$ time points). The profiles resulting from these analyses were used to get the net plasma membrane recruitment profiles (Fig S1A, Lamas et al. 2020), the fluorescence intensities from the peak \pm 1 pixel were averaged and plotted over time.

(3) Peak RFP intensity $_{\text {tn }}=\left(\right.$ RFP intensity peak-1pixel tn $+R F P$ intensity $_{\text {peak } t n}+R F P$ intensity $\left._{\text {peak }+1 p i x e l ~ t n}\right) / 3$

(4) Net P.M.recruitment Profile $=\left(\right.$ Peak RFP intensity tn - Peak RFP intensity $\left.{ }_{t 0}\right)$

Finally, the single-cell plasma membrane recruitment half-times were calculated by fitting the normalized recruitment profiles with the following formula:

(5) RFP intensity $(y)=a *\left(1-e^{(-b * t)}\right)$

(6) Recruitment $t_{1 / 2}=\ln (0.5) / \mathrm{b}$

\section{Quantifications of the re-localization of GFP-tagged proteins to cell sides:}

Endogenous GFP-tagged protein re-localization was assessed upon photo-activation of CRY2$\mathrm{Cdc}_{2} 2^{\Delta \mathrm{CaaX}}$ and CRY2_systems by recording the fluorescence intensity over a 3 pixel-wide by 36 pixellong $(\approx 0.25 \mu \mathrm{m}$ by $3 \mu \mathrm{m}) \mathrm{ROI}$ drawn parallel to the cell side cortex of sample cells. The average 
fluorescence intensity values of both GFP and RFP channels were recorded over time from sample strains. In these particular experiments, a GFP control strain was included. These strains serve 2 purposes:

- $\quad$ Calculation of the GFP bleaching correction coefficient (see below).

- $\quad$ Negative control of the experiment. These strains carry the same endogenous GFP-tagged protein as the sample strain of the experiment, however lacking the optogenetic system. This controlled that GFP fluorescence changes were due to the optogenetic system and not caused by imaging per se. Control GFP strains were imaged in the same pad and analyzed in the same way as optogenetic cells.

To derive photobleaching correction coefficients, the average camera background signals $(B c k g)$ from 5 cell-free regions was measured as above, and fluorophore bleaching from RFP control and GFP control strains were measured at the cell side of control RFP and control GFP strains, for RFP and GFP channels, respectively.

$$
\begin{aligned}
& \text { (7) RFP bleaching correction coefficient }=\left(R F P \text { Intensity } y_{t n}-B c k g_{t n}\right) /\left(R F P \text { Intensity } y_{t 0}-B c k g_{t 0}\right) \\
& \text { (8) GFP bleaching correction coefficient }=\left(G F P \text { Intensity } y_{t n}-B c k g_{t n}\right) /\left(G F P \text { Intensity } y_{t 0}-B c k g_{t 0}\right)
\end{aligned}
$$

where RFP intensity and GFP intensity stand for the signal measured from RFP control and GFP control cells, respectively, $t_{n}$ represents a given time point along the time course of the experiment and $t_{0}$ represents the initial time point $(n=30$ time points). These coefficients were corrected by a moving average smoothing method, as above.

The fluorescence intensity values of optogenetic cells in both GFP and RFP channels were independently analyzed as follows. First, GFP and RFP signals were background and bleaching corrected, using formulas (7) and (8) for the RFP and GFP channels, respectively:

(9) P.M.GFP/RFP value tn $_{n}=\left(\left(\right.\right.$ Raw signal $_{t n}-$ Bckg $\left._{t n}\right) /$ bleaching correction coefficient $\left._{t n}\right)$

where Raw signal intensity represents the GFP or RFP raw values at the cell side cortex, Bckg stands for the average fluorescence intensity of 5 independent cell-free regions and $t_{n}$ represents a given time point along the time course of the experiment ( $n=30$ time points). The net fluorescence intensity at the cell side cortex was then calculated for both GFP and RFP signals.

(10) Net P.M.GFP/RFP value tn $_{1}=$ (Fluorescence intensity trn $_{-}-$Fluorescence intensity $_{t 0}$ )

From here on, RFP and GFP signals were treated differently. Single cell plasma membrane RFP profiles from equation (10) were individually normalized and fitted to the equation (5) in order to extrapolate the parameter b. Using the equation (6), recruitment half times of CRY2 and CRY2-Cdc42 ${ }^{\triangle \mathrm{CaaX}}$ were calculated. Because of lower signal-to-noise of the weak GFP fluorescence, plasma membrane GFP profiles from equation (10) were averaged ( $n>20$ profiles per experiment), and the initial $45 \mathrm{~s}$ of the 
average profile used to extract the half-time of plasma membrane re-localization of endogenous GFPtagged proteins using equations (5) and (6). 3 experimental replicates were performed and are plotted on Fig 1B; Fig 2B-D, Fig 3B-E-G.

\section{Quantifications of the re-localization of GFP-tagged proteins from cell tips:}

Scd1-3GFP tip signal analyses (Fig 3C-F) were performed from the same time-lapse recordings as cell side re-localization experiments. Scd1-3GFP tip signal was recorded over a 3 pixel-wide by 6-12 pixellong $(\approx 0.25 \mu \mathrm{m}$ by $0.5-1 \mu \mathrm{m}) \mathrm{ROI}$ drawn at the tip of the cells. To derive photobleaching correction coefficients, the average camera background signals $(B c k g)$ from 5 cell-free regions was measured as before, and GFP bleaching from GFP control strain was measured at the cell tip.

(11) Tip GFP bleaching correction coefficient $=\left(G F P\right.$ Intensity $\left.y_{t n}-B c k g_{t n}\right) /\left(G F P\right.$ Intensity $\left.y_{t 0}-B c k g_{t 0}\right)$

where GFP intensity stands for the signal measured from the tip of GFP control cells, $t_{n}$ represents a given time point along the time course of the experiment and to represents the initial time point $(n=30$ time points). This coefficient was corrected by a moving average smoothing method, as before.

The tip GFP fluorescence intensity values of optogenetic cells was analyzed as follows. First, GFP signals was background and bleaching corrected, using formula (12):

\left. (12) ${\text { Tip GFP } \text { value }_{t n}=(\text { Tip Raw signal }}_{t n}-B c k g_{t n}\right) /$ Tip GFP bleaching correction coef ficient $t_{t n}$

where Tip Raw signal intensity represents the GFP raw values at the cell tip, Bckg stands for the average fluorescence intensity of 5 independent cell-free regions and $t_{n}$ represents a given time point along the time course of the experiment ( $n=30$ time points). The tip fluorescence intensities of single optogenetic strains were then normalized relative to their GFP values at the initial time-point.

(13) Normalized tip GFP value $_{t n}=\left(\right.$ Tip GFP value $_{t n} /{\left.\text { tip } G F P_{t 0}\right)}$

Eventually, average Scd1-3GFP tip signal was calculated (>15 cells, Fig 3C-F).

\section{Quantifications of CRIB-3GFP and Cdc42-mCh ${ }^{\text {sw }}$ relative expression and distribution profiles:}

CRIB-3GFP fluorescence intensity was measured from sum projection of 6 Z-stacks (Fig S2B). The background signal from cell-free regions were used to correct the data. The relative fluorescence intensities were calculated by dividing the single-cell CRIB-3GFP fluorescence intensity measurements of WT and Pact1-cdc42-mCh ${ }^{\text {SW }}$ cells by the average CRIB-3GFP fluorescence intensity of WT cells.

CRIB-3GFP distribution profiles were generated from sum projection images of 5 middle-sections (Fig S2C). 3-pixel wide ROls were drawn from side to side following cell membrane contour. The background 
signal from cell-free regions were used to correct the data. Whole tip profiles were split in half based on the pixel position of their maximum CRIB-3GFP intensity (approximately in the middle of the profile), generating 60 half tips. To take into account the CRIB-3GFP expression level, the average CRIB-3GFP profiles from WT and Pact1-cdc42- $m C h^{s W}$ cells were then normalized by dividing each value by the relative CRIB-3GFP fluorescence intensities values shown in Figure S2B.

\section{Cell size measurements, aspect ratios and index of ratios:}

The aspect ratio of mutant cells was calculated by dividing the cell length by the cell width (Fig 4A). In Fig 4B, the index of ratios were calculated by dividing CRY2 control aspect ratios by CRY2-Cdc42 ${ }^{\mathrm{ACaaX}}$ and CRY2-Cdc42 ${ }^{\mathrm{Q} 61 \mathrm{~L}, \mathrm{C}} \mathrm{CaaX}$.

Figures were assembled with Adobe Photoshop CS5 and Adobe Illustrator CS5. All error bars on bar graphs are standard deviations. For statistical analysis, in Fig 1, 2 and 3, cumulative GFP signal (addition of GFP signal along the 30 time points of the time-lapse) was calculated from single cell traces of CRY2, CRY2-Cdc42 ${ }^{\triangle \mathrm{Caax}}$ and GFP control cells. For statistical analysis, single cell cumulative GFP signals of the entire dataset ( 3 independent experiments combined) were considered, without averaging. Data normality was assessed by the Lilliesfors test and significance by pair-wise KruskalWallis analysis. $P$ values show significance of differences between CRY2-Cdc42 ${ }^{\triangle \mathrm{CaaX}}$ and CRY2 cells, unless indicated otherwise. T-test was used in Fig 4 . On each box the central red mark indicates the median, while the bottom and the top edges indicate the $25^{\text {th }}$ and $75^{\text {th }}$ percentiles respectively, the whiskers extend to the most extreme data points not considering outliers, which are plotted individually using the red ' + ' symbol. All experiments were done at least three independent times.

\section{Acknowledgements}

We thank Dr Aleksandar Vjestica (University of Lausanne) for gift of the CRY2-Cdc42 ${ }^{\Delta \mathrm{CaaX}}$ strain and Dr Serge Pelet (University of Lausanne) for help with MatLab. This work was funded by ERC Consolidator grant (CellFusion) to SGM.

\section{Author contributions}

IL and SGM conceived the project. NW performed the experiments presented in Fig 4. IL performed all other experiments. IL and SGM wrote the manuscript. SGM acquired funding.

\section{$\underline{\text { References }}$}

Bähler, J., J.Q. Wu, M.S. Longtine, N.G. Shah, A. McKenzie, 3rd, A.B. Steever, A. Wach, P. Philippsen, and J.R. Pringle. 1998. Heterologous modules for efficient and versatile PCR-based gene targeting in Schizosaccharomyces pombe. Yeast. 14:943-951. 
Bendezu, F.O., and S.G. Martin. 2011. Actin cables and the exocyst form two independent morphogenesis pathways in the fission yeast. Mol Biol Cell. 22:44-53.

Bendezu, F.O., V. Vincenzetti, D. Vavylonis, R. Wyss, H. Vogel, and S.G. Martin. 2015. Spontaneous Cdc42 polarization independent of GDI-mediated extraction and actin-based trafficking. PLoS Biol. 13:e1002097.

Bugaj, L.J., A.T. Choksi, C.K. Mesuda, R.S. Kane, and D.V. Schaffer. 2013. Optogenetic protein clustering and signaling activation in mammalian cells. Nat Methods. 10:249-252.

Chang, E.C., M. Barr, Y. Wang, V. Jung, H.P. Xu, and M.H. Wigler. 1994. Cooperative interaction of S. pombe proteins required for mating and morphogenesis. Cell. 79:131-141.

Coll, P.M., Y. Trillo, A. Ametzazurra, and P. Perez. 2003. Gef1p, a New Guanine Nucleotide Exchange Factor for Cdc42p, Regulates Polarity in Schizosaccharomyces pombe. Mol Biol Cell. 14:313323.

Das, M., D.J. Wiley, S. Medina, H.A. Vincent, M. Larrea, A. Oriolo, and F. Verde. 2007. Regulation of cell diameter, For3p localization, and cell symmetry by fission yeast Rho-GAP Rga4p. Mol Biol Cell. 18:2090-2101.

Dudin, O., F.O. Bendezu, R. Groux, T. Laroche, A. Seitz, and S.G. Martin. 2015. A formin-nucleated actin aster concentrates cell wall hydrolases for cell fusion in fission yeast. J Cell Biol. 208:897911.

Endo, M., M. Shirouzu, and S. Yokoyama. 2003. The Cdc42 binding and scaffolding activities of the fission yeast adaptor protein Scd2. J Biol Chem. 278:843-852.

Gallo Castro, D., and S.G. Martin. 2018. Differential GAP requirement for Cdc42-GTP polarization during proliferation and sexual reproduction. J Cell Biol. 217:4215-4229.

Hirota, K., K. Tanaka, K. Ohta, and M. Yamamoto. 2003. Gef1p and Scd1p, the Two GDP-GTP exchange factors for Cdc42p, form a ring structure that shrinks during cytokinesis in Schizosaccharomyces pombe. Mol Biol Cell. 14:3617-3627.

Inouye, K., S. Mizutani, H. Koide, and Y. Kaziro. 2000. Formation of the Ras dimer is essential for Raf1 activation. J Biol Chem. 275:3737-3740.

Kang, P.J., L. Beven, S. Hariharan, and H.O. Park. 2010. The Rsr1/Bud1 GTPase interacts with itself and the Cdc42 GTPase during bud-site selection and polarity establishment in budding yeast. Mol Biol Cell. 21:3007-3016.

Kelly, F.D., and P. Nurse. 2011. Spatial control of Cdc42 activation determines cell width in fission yeast. Mol Biol Cell. 22:3801-3811.

Kennedy, M.J., R.M. Hughes, L.A. Peteya, J.W. Schwartz, M.D. Ehlers, and C.L. Tucker. 2010. Rapid blue-light-mediated induction of protein interactions in living cells. Nat Methods. 7:973-975.

Lamas, I., L. Merlini, A. Vjestica, V. Vincenzetti, and S.G. Martin. 2020. Optogenetics reveals Cdc42 local activation by scaffold-mediated positive feedback and Ras GTPase. PLoS Biol. 18:e3000600.

Maxwell, K.N., Y. Zhou, and J.F. Hancock. 2018. Rac1 Nanoscale Organization on the Plasma Membrane Is Driven by Lipid Binding Specificity Encoded in the Membrane Anchor. Mol Cell Biol. 38.

Meca, J., A. Massoni-Laporte, D. Martinez, E. Sartorel, A. Loquet, B. Habenstein, and D. McCusker. 2019. Avidity-driven polarity establishment via multivalent lipid-GTPase module interactions. EMBO J. 38.

Merlini, L., B. Khalili, F.O. Bendezu, D. Hurwitz, V. Vincenzetti, D. Vavylonis, and S.G. Martin. 2016. Local Pheromone Release from Dynamic Polarity Sites Underlies Cell-Cell Pairing during Yeast Mating. Curr Biol. 26:1117-1125.

Merlini, L., B. Khalili, O. Dudin, L. Michon, V. Vincenzetti, and S.G. Martin. 2018. Inhibition of Ras activity coordinates cell fusion with cell-cell contact during yeast mating. J Cell Biol. 217:1467-1483.

Nakano, K., T. Mutoh, R. Arai, and I. Mabuchi. 2003. The small GTPase Rho4 is involved in controlling cell morphology and septation in fission yeast. Genes Cells. 8:357-370.

Nussinov, R., C.J. Tsai, and H. Jang. 2020. Ras assemblies and signaling at the membrane. Curr Opin Struct Biol. 62:140-148.

Remorino, A., S. De Beco, F. Cayrac, F. Di Federico, G. Cornilleau, A. Gautreau, M.C. Parrini, J.B. Masson, M. Dahan, and M. Coppey. 2017. Gradients of Rac1 Nanoclusters Support Spatial Patterns of Rac1 Signaling. Cell reports. 21:1922-1935.

Revilla-Guarinos, M.T., R. Martin-Garcia, M.A. Villar-Tajadura, M. Estravis, P.M. Coll, and P. Perez. 2016. Rga6 is a Fission Yeast Rho GAP Involved in Cdc42 Regulation of Polarized Growth. Mol Biol Cell. 
Sartorel, E., C. Unlu, M. Jose, A. Massoni-Laporte, J. Meca, J.B. Sibarita, and D. McCusker. 2018. Phosphatidylserine and GTPase activation control Cdc42 nanoclustering to counter dissipative diffusion. Mol Biol Cell. 29:1299-1310.

Tatebe, H., K. Nakano, R. Maximo, and K. Shiozaki. 2008. Pom1 DYRK regulates localization of the Rga4 GAP to ensure bipolar activation of Cdc42 in fission yeast. Curr Biol. 18:322-330.

Tay, Y.D., M. Leda, A.B. Goryachev, and K.E. Sawin. 2018. Local and global Cdc42 GEFs for fission yeast cell polarity are coordinated by microtubules and the Tea1/Tea4/Pom1 axis. J Cell Sci.

Vjestica, A., D. Zhang, J. Liu, and S. Oliferenko. 2013. Hsp70-Hsp40 chaperone complex functions in controlling polarized growth by repressing Hsf1-driven heat stress-associated transcription. PLoS genetics. 9:e1003886.

Wheatley, E., and K. Rittinger. 2005. Interactions between Cdc42 and the scaffold protein Scd2: requirement of $\mathrm{SH} 3$ domains for GTPase binding. Biochem J. 388:177-184.

Zhang, B., Y. Gao, S.Y. Moon, Y. Zhang, and Y. Zheng. 2001. Oligomerization of Rac1 gtpase mediated by the carboxyl-terminal polybasic domain. J Biol Chem. 276:8958-8967.

Zhang, B., and Y. Zheng. 1998. Negative regulation of Rho family GTPases Cdc42 and Rac2 by homodimer formation. J Biol Chem. 273:25728-25733.

\section{Figure legends}

Figure 1. Ectopic sites of Cdc42 activation upon CRY2-Cdc42 ${ }^{\triangle \mathrm{CaaX}}$ cell-sides recruitment. $(\mathrm{A})$ Localization of Scd2-GFP, CRIB-3GFP and Pak1-sfGFP in CRY2-Cdc42 ${ }^{\triangle C a a X}$-expressing cells (B/W inverted images). The GFP max projection ("max proj.") images show GFP maximum-intensity projections of 30 time points over $90 \mathrm{~s}$ and illustrate best the side recruitment of GFP-tagged probes. The GFP max projection 60 - $90 \mathrm{~s}$ ("max proj. 60 - $90 \mathrm{~s}$ ") images show GFP maximum-intensity projections of 10 last time points over $30 \mathrm{~s}$. Orange arrowheads point to lateral CRIB-3GFP signal. Pink arrowheads point to Scd2-GFP tip signal decrease. RFP images show the cortical recruitment of CRY2$\mathrm{Cdc} 42^{\triangle \mathrm{CaaX}}$ and CRY2 at the end of the time lapse. The internal globular and filamentous signal is due to cellular autofluorescence. (B) Quantification of GFP signal intensities of Scd2-GFP, CRIB-3GFP and Pak1-sfGFP at cell sides in WT cells. $N=3 ; n>20$ cells per experiment; $p^{\text {Scd2-GFP }}=1.55 \mathrm{e}^{-8} ; p^{\text {CRIB-3GFP }}$ $=0.019 ; p^{\text {Pak1-sfGFP }}=0.168$. $(\mathrm{C})$ Comparison of data from panel $(\mathrm{B})$ with $\mathrm{CRY} 2-\mathrm{Cdc} 42^{\mathrm{Q} 61 \mathrm{~L}, \Delta \mathrm{CaaX}}$-induced cell-side re-localization of Scd2-GFP, CRIB-3GFP and Pak1-sfGFP (data from (Lamas et al., 2020)). In all graphs, thick line = average; shaded area = standard deviation; WT, wild type; A.U., arbitrary units. Bars $=2 \mu \mathrm{m}$. Associated trace analysis is shown in Fig S3A.

Figure 2. Role of Cdc42 GEFs in activation of CRY2-Cdc42 ${ }^{\triangle \mathrm{Caax}}$. (A) Localization of Scd2-GFP, CRIB-3GFP and Pak1-sfGFP in CRY2-Cdc42 ${ }^{\triangle \mathrm{CaaX}}$-expressing scd1 $\Delta$ cells (B/W inverted images). The GFP max projection ("max proj.") images show GFP maximum-intensity projections of 30 time points over $90 \mathrm{~s}$ and illustrate best the side recruitment of GFP-tagged probes. The GFP max projection $60-$ $90 \mathrm{~s}$ ("max proj. 60 - $90 \mathrm{~s}$ ") images show GFP maximum-intensity projections of 10 last time points over $30 \mathrm{~s}$. Orange arrowheads point to lateral CRIB-3GFP and Scd2-GFP signal. RFP images show the cortical recruitment of CRY2-Cdc42 ${ }^{\triangle \mathrm{Caax}}$ and CRY2 at the end of the time lapse. The internal globular and filamentous signal is due to cellular autofluorescence. (B) Quantification of GFP signal intensities of CRIB-3GFP, Pak1-sfGFP and Scd2-GFP at cell sides of $s c d 1 \Delta$ mutants. $N=3 ; n>20$ cells per 
experiment; $p^{\text {Pak1-sfGFP }}=0.172 ; p^{\text {CRIB-3GFP }}=0.119 ; p^{\text {Scd2-GFP }}=2.49 e^{-9} .(C)$ Localization of Scd2-GFP in CRY2-Cdc42 ${ }^{\Delta \mathrm{CaaX}}$-expressing gef1 $\Delta$ cells (B/W inverted images). Arrangement of images as in panel A. (D) Quantification of GFP signal intensity of Scd2-GFP at cell sides of gef1 $\Delta$ mutants. $N=3 ; n>20$ cells per experiment; $p^{\text {Scd2-GFP }}=2.82 \mathrm{e}^{-4}$. In all graphs, thick line $=$ average; shaded area $=$ standard deviation. Bars $=2 \mu \mathrm{m}$. A.U., arbitrary units; WT, wild type. Associated trace analysis is shown in Fig S3B-C.

Figure 3. CRY2-Cdc42 ${ }^{\triangle \mathrm{CaaX}}$ recruits the GEF Scd1 dependent on the scaffold Scd2. (A) Localization of Scd1-3GFP in CRY2-Cdc42 ${ }^{\triangle \mathrm{CaaX}}$-expressing cells (B/W inverted images). The GFP max projection ("max proj.") images show GFP maximum-intensity projections of 30 time points over $90 \mathrm{~s}$ and illustrate best the side recruitment of GFP-tagged probes. The GFP max projection 60 - $90 \mathrm{~s}$ ("max proj. 60 - $90 \mathrm{~s}$ ") images show GFP maximum-intensity projections of 10 last time points over $30 \mathrm{~s}$. Orange arrowheads point to lateral Scd1-3GFP signal. Pink arrowheads point to Scd1-3GFP tip signal decrease. RFP images show the cortical recruitment of CRY2-Cdc42 ${ }^{\triangle \mathrm{CaaX}}$ and CRY2 at the end of the time lapse. The internal globular and filamentous signal is due to cellular autofluorescence. (B) Quantification of GFP signal intensity of Scd1-3GFP at cell sides of WT cells. N = 3; $n>20$ cells per experiment; $p^{\text {Scd1-3GFP }}=0.012$. (C) Quantification of GFP signal intensity of Scd1-3GFP at cell poles of WT cells expressing CRY2-Cdc42 ${ }^{\Delta C a a X}$ or CRY2 $(p=2.14 \mathrm{e}-4)$. $N=3 ; n>15$ cells per experiment. (D) Localization of Scd1-3GFP and CRIB-3GFP in CRY2-Cdc42 ${ }^{\triangle \mathrm{Caax}}$-expressing scd2 $\Delta$ cells (B/W inverted images). Arrangement of images as in panel A. Orange arrowheads point to lateral CRIB-3GFP signal. The internal globular and filamentous signal is due to cellular autofluorescence. (E) Quantification of Scd1-3GFP at cell sides of $s c d 2 \Delta$ mutant. $N=3 ; n>20$ cells per experiment; $p^{\text {scd1-3GFP }}=0.386$. $(\mathrm{F})$ Quantification of Scd1-3GFP at cell poles of $s c d 2 \Delta$ mutants expressing CRY2-Cdc42 ${ }^{\Delta C a a x}$ or CRY2 ( $p$ $=0.37$ ). $\mathrm{N}=3 ; \mathrm{n}>15$ cells. (G) Quantification of CRIB-3GFP at cell sides of $s c d 2 \Delta$ mutants. $N=3 ; n>$ 20 cells per experiment; $p^{\text {CRIB-3GFP }}=7.67 \mathrm{e}^{-4}$. $(\mathrm{H})$ Comparison of cumulative CRIB-3GFP intensities in

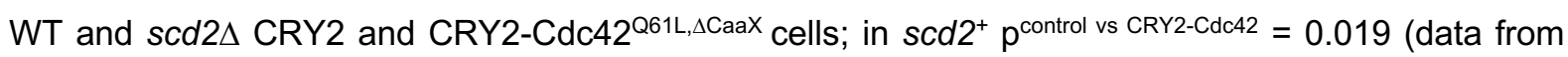

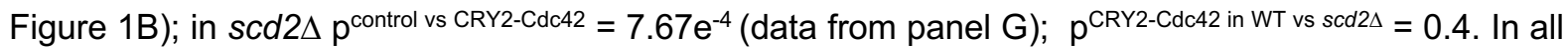
graphs, thick line $=$ average; shaded area $=$ standard deviation. Bars $=2 \mu \mathrm{m}$. A.U., arbitrary units; WT, wild type. Associated trace analysis is shown in Fig S4.

Figure 4. Rga4 GAP prevents growth on cell sides in CRY2-Cdc42 ${ }^{\Delta \mathrm{Caa}}$ cells. (A) Average cell length $(\mu \mathrm{m})$, cell width $(\mu \mathrm{m})$ and aspect ratio of CRY2, CRY2-Cdc42 ${ }^{\triangle \mathrm{Caax}}$ and CRY2-Cdc42 ${ }^{\mathrm{Q} 61 \mathrm{~L}, \triangle \mathrm{CaaX}}$ mutants. For all mutant $\mathrm{N}=2, \mathrm{n}>80$ cells per experiment; except for gap1 $\Delta$ mutants, $\mathrm{N}=1, \mathrm{n}>80$ cells per experiment. (B) Aspect ratios of CRY2-Cdc42 ${ }^{\triangle \mathrm{Caax}}$ and CRY2-Cdc42 ${ }^{\mathrm{Q} 61 \mathrm{~L}, \Delta \mathrm{Caax}}$-expressing cells grown in the light normalized to the aspect ratios of CRY2-expressing cells for all the tested mutants. Bars = standard error. The green background indicates expected reduction in aspect ratio upon CRY2Cdc42 ${ }^{\mathrm{Q} 61 \mathrm{~L}, \triangle \mathrm{CaaX}}$ recruitment in WT cells. The blue background indicates absence of change in aspect ratio upon $\mathrm{CRY} 2-\mathrm{Cdc} 42^{\mathrm{Q} 61 \mathrm{~L}}$ recruitment. Note that all rga4 $\Delta$ mutants fall in the white intermediate space.

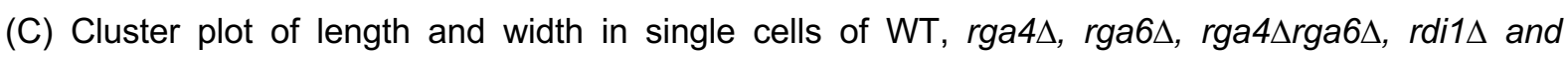




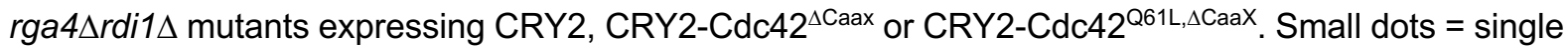
cells; Large, dark dots = average; bars = standard deviation; A.U., arbitrary units; WT, wild type.

Figure S1. Controls for CRY2-Cdc42 ${ }^{\triangle \mathrm{CaaX}}$ optogenetic recruitment. (A) Plasma membrane recruitment dynamics of CRY2 and CRY2-Cdc42 ${ }^{\triangle \mathrm{CaaX}}$ in response to periodic $50 \mathrm{~ms}$ (top), $250 \mathrm{~ms}$ (middle) and $500 \mathrm{~ms}$ (bottom) blue-light $(\lambda=485 \mathrm{~nm})$ pulses $(\mathrm{N}=3 ; \mathrm{n}=30$ cells per experiment). Thick line = average; shaded area = standard deviation. CRY2 values are from (Lamas et al., 2020). (B) Plasma-membrane recruitment half-times for CRY2 and CRY2-Cdc42 ${ }^{\triangle \mathrm{Caax}}$. On each box, the central mark indicates the median; the bottom and the top edges indicate the 25th and 75th percentiles, respectively; the whiskers extend to the most extreme data points not considering outliers, which are plotted individually using the red "+" symbol. (C) Cell length and width measurements, aspect ratio and bipolarity of calcofluor-stained cells growing in the dark. CRY2 and CRY2-Cdc42 ${ }^{\Delta \mathrm{CaaX}}$ do not have any effect on cell morphology $\left(p^{\text {WTvsCRY2 }}=0.19 ; p^{\text {WTvsCRY2-Cdc42 }}=0.19 ; p^{\text {CRY2vsCRY2-Cdc42 }}=0.83\right.$ ). Monopolarity and bipolarity were assessed on septating cells. (D) Blue light-dependent induction of isotropic growth in CRY2-Cdc42 ${ }^{\mathrm{Q} 61 L \Delta \mathrm{CaaX}}$ (blue cells), but not CRY2-Cdc42 ${ }^{\Delta \mathrm{CaaX}}$ (green cells) cells photoactivated at 10min interval (GFP, RFP, and BF channels were acquired every $10 \mathrm{~min}$; UV channel every $1 \mathrm{~h}$ ). Note that the patchy appearance of CRY2 is likely due to the clustering properties of this protein. Bars $=5$ $\mu \mathrm{m}$.

Figure S2. Overexpression of $\mathrm{Cdc42-} \mathrm{mCh}^{\mathrm{sW}}$ in WT cells does not induce ectopic activation of Cdc42. (A) $p^{\text {act1 }}$ promoter induces overexpression of $\mathrm{Cdc42-mCh} \mathrm{CW}^{\mathrm{sW}}$ in CRIB-3GFP cells. Two different contrasting settings are shown. (B) Relative expression of CRIB-3GFP and Cdc42-mCh ${ }^{\text {SW }}$ in WT (cdc42-mCherry ${ }^{s W}$ ) and $p^{\text {act1 }}$-cdc42-mCherrysw cells. $p^{\text {act1 }}$-cdc42 cells exhibit $1.2 \mathrm{x}$ and $3.3 \mathrm{x}$ higher CRIB-3GFP and Cdc42-mCh ${ }^{\text {SW }}$ fluorescence intensities respectively as compared to WT cells. $N=1$; $n=50$ cells. (C) Distribution profile of CRIB-3GFP in WT and $p^{\text {act1 }}$-cdc42 cells at the cortex from the centre of cell tips towards the cell side. Blue area represents the centre of the tip $(0.5 \mu \mathrm{m})$ and red area represents the cell side. The CRIB profile was corrected by the 1.2-fold increase in CRIB expression. (D) CRIB-3GFP fluorescence intensities at the cell tip (left) and cell sides (right) in WT (cdc42$m$ Cherry ${ }^{s w}$ ) and $p^{\text {act } 1}-c d c 42-m C h e r r y{ }^{s w}$ cells derived from the blue and red areas shown in C. $\mathrm{p}^{\text {CRIB- }}$ ${ }_{3 \text { GFP-Tip }}=3.7 \mathrm{e}-5 ; \mathrm{p}^{\text {CRIB-3GFP-Side }}=0.18 . N=1 ; n=60$ half tips. In all graphs, thick line = average; bars \& shaded area $=$ standard deviation; WT, wild type; A.U., arbitrary units. Bars $=5 \mu \mathrm{m}$.

Figure S3. Control and single-cell traces for optogenetic recruitment in WT, scd1 $\Delta$ and gef1 $\triangle$ cells. (A) Single-cell traces corresponding to the average plots shown in Fig 1B. The left column shows the average RFP signal at the plasma membrane of wildtype CRY2-Cdc42 ${ }^{\triangle \mathrm{CaaX}}$ and CRY2 cells. The three other graphs show, from left to right, single cell GFP traces of CRY2-Cdc42 ${ }^{\triangle C a a x}, C R Y 2$ and WT control cells for Scd2-GFP, CRIB-3GFP and Pak1-sfGFP in otherwise wildtype cells. (B) Single-cell traces corresponding to the average plots shown in Fig 3B. The left column shows the average RFP signal at the plasma membrane of wildtype CRY2-Cdc42 ${ }^{\triangle \mathrm{Caax}}$ and CRY2 cells. The three other graphs 
show, from left to right, single cell GFP traces of CRY2-Cdc42 ${ }^{\Delta \mathrm{Caax}}, \mathrm{CRY} 2$ and WT control cells for CRIB-3GFP, Pak1-sfGFP and Scd2-GFP in scd1 $\Delta$ mutants. (C) Single-cell traces corresponding to the average plots shown in Fig 3D. The left column shows the average RFP signal at the plasma membrane of wildtype CRY2-Cdc42 ${ }^{\triangle \mathrm{CaaX}}$ and CRY2 cells. The three other graphs show, from left to right, single cell GFP traces of CRY2-Cdc42 ${ }^{\triangle \mathrm{CaaX}}, \mathrm{CRY} 2$ and WT control cells for Scd2-GFP in gef1 $\Delta$ mutants.

Figure S4. Control and single-cell traces for optogenetic recruitment of Scd1-3GFP and CRIB3GFP in WT and sdc2 $\triangle$ cells. A) Single-cell traces corresponding to the average plots shown in Fig 4B. The left column shows the average RFP signal at the plasma membrane of wildtype CRY2Cdc42 ${ }^{\triangle \mathrm{CaaX}}$ and CRY2 cells. The three other graphs show, from left to right, single cell GFP traces of CRY2-Cdc42 ${ }^{\triangle \mathrm{Caa}}, \mathrm{CRY} 2$ and WT control cells for Scd1-3GFP in otherwise wildtype cells. (B) Singlecell traces corresponding to the average plots shown in Fig $4 \mathrm{C}$. The three graphs show, from left to right, single cell GFP traces of CRY2-Cdc42 ${ }^{\triangle \mathrm{Caax}}, \mathrm{CRY} 2$ and WT control cells for normalized tip signal of Scd1-3GFP in otherwise wildtype cells. (C) Single-cell traces corresponding to the average plots shown in Fig 4E-G. The left column shows the average RFP signal at the plasma membrane of wildtype CRY2-Cdc42 ${ }^{\triangle \mathrm{CaaX}}$ and CRY2 cells. The three other graphs show, from left to right, single cell GFP traces of CRY2-Cdc42 ${ }^{\triangle \mathrm{CaaX}}, \mathrm{CRY} 2$ and WT control cells for Scd1-3GFP and CRIB-3GFP in scd2 $\Delta$ mutants. (D) Single-cell traces corresponding to the average plots shown in Fig 4F. The three graphs show, from left to right, single cell GFP traces of CRY2-Cdc42 ${ }^{\triangle \mathrm{Caax}}, \mathrm{CRY} 2$ and WT control cells for normalized tip signal of Scd1-3GFP in $s c d 2 \Delta$ mutants.

\section{Table S1: List of strains used in this study.}


bioRxiv preprint doi: https://doi.org/10.1101/2020.06.02.130336; this version posted June 2, 2020. The copyright holder for this preprint Figure 1 (which was not certified by peer review) is the author/funder, who has granted bioRxiv a license to display the preprint in perpetuity. It is
made available under aCC-BY-NC 4.0 International license.

A

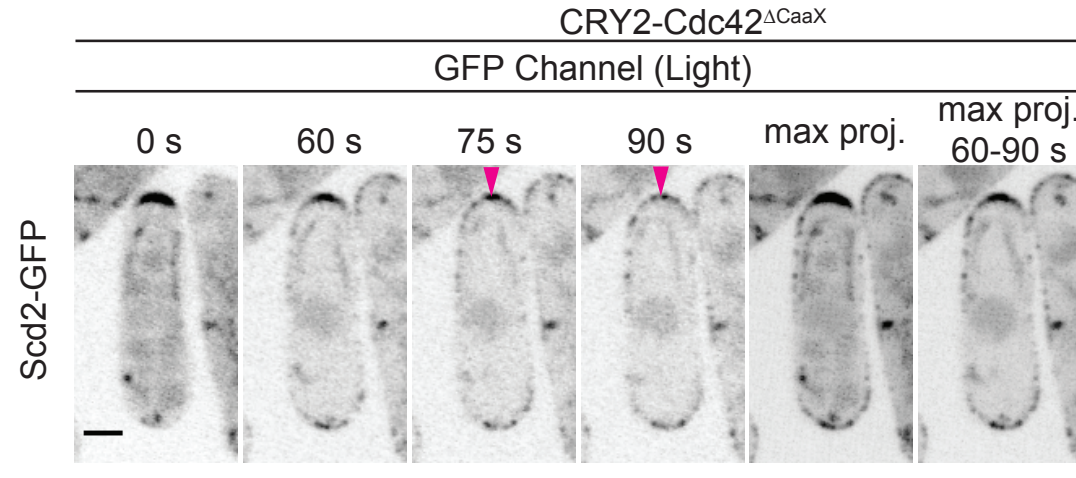

$\frac{\text { CRY2 Control }}{\text { GFP }}$

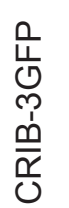
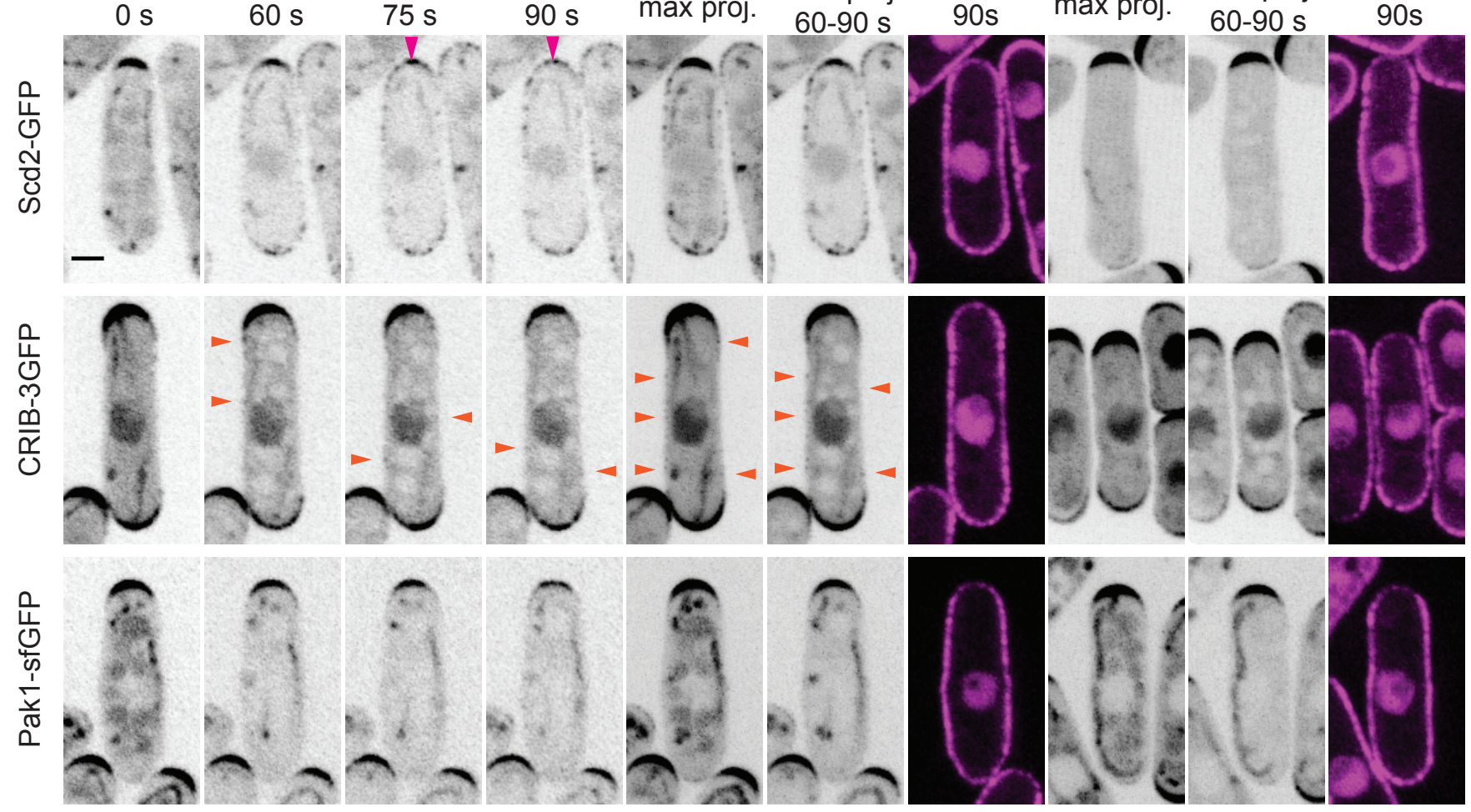

B
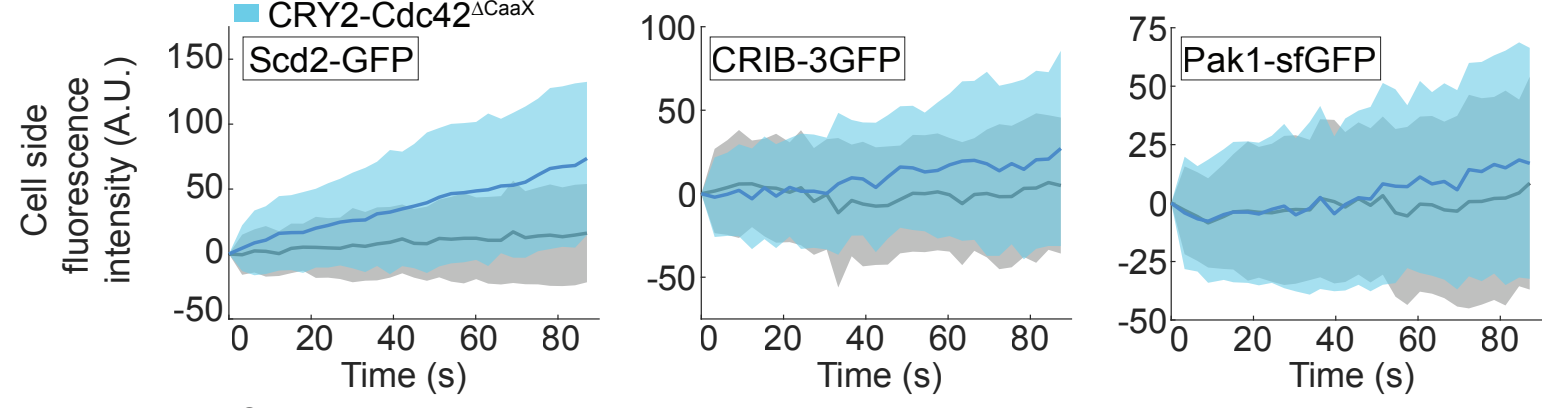

C

$$
\begin{aligned}
& \text { CRY2 control } \\
& \text { CRY2-Cdc42 }{ }^{\triangle \mathrm{Caax}} \\
& \text { CRY2-Cdc42 }
\end{aligned}
$$
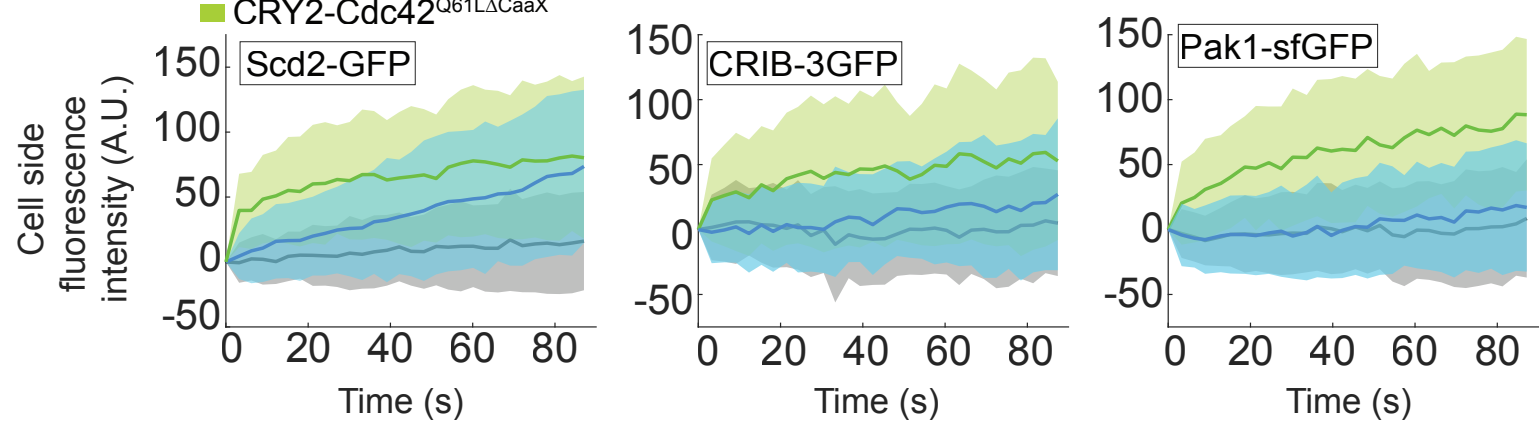
bioRxiv preprint doi: https://doi.org/10.1101/2020.06.02 130336. this version posted June 2 2020. The copyriaht holder for this preprint Figure 2which was not certified by peer review) is the author/funder, who has granted bioRxiv a license to display the preprint in perpetuity. It is made available under aCC-BY-NC 4.0 International license.

A

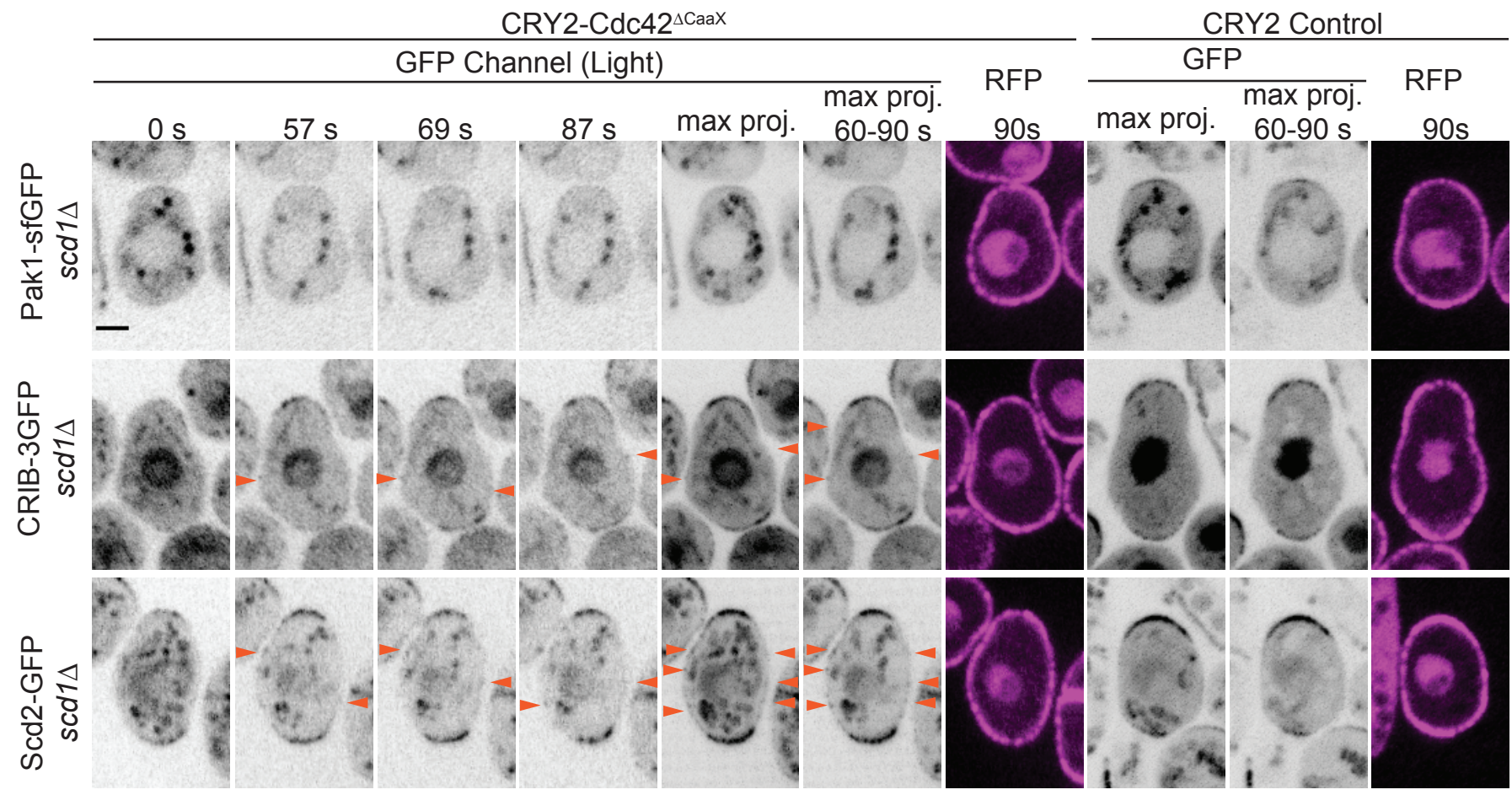

B

$\operatorname{scd} 1 \Delta$ CRY2 control $\quad s c d 1 \Delta$ CRY2-Cdc42 ${ }^{\Delta C a a X}$
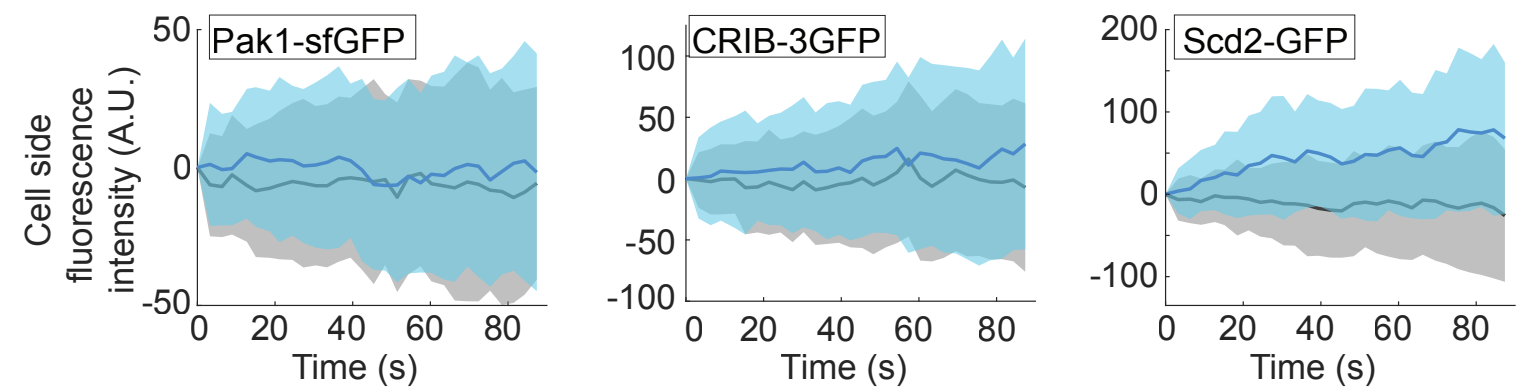

C
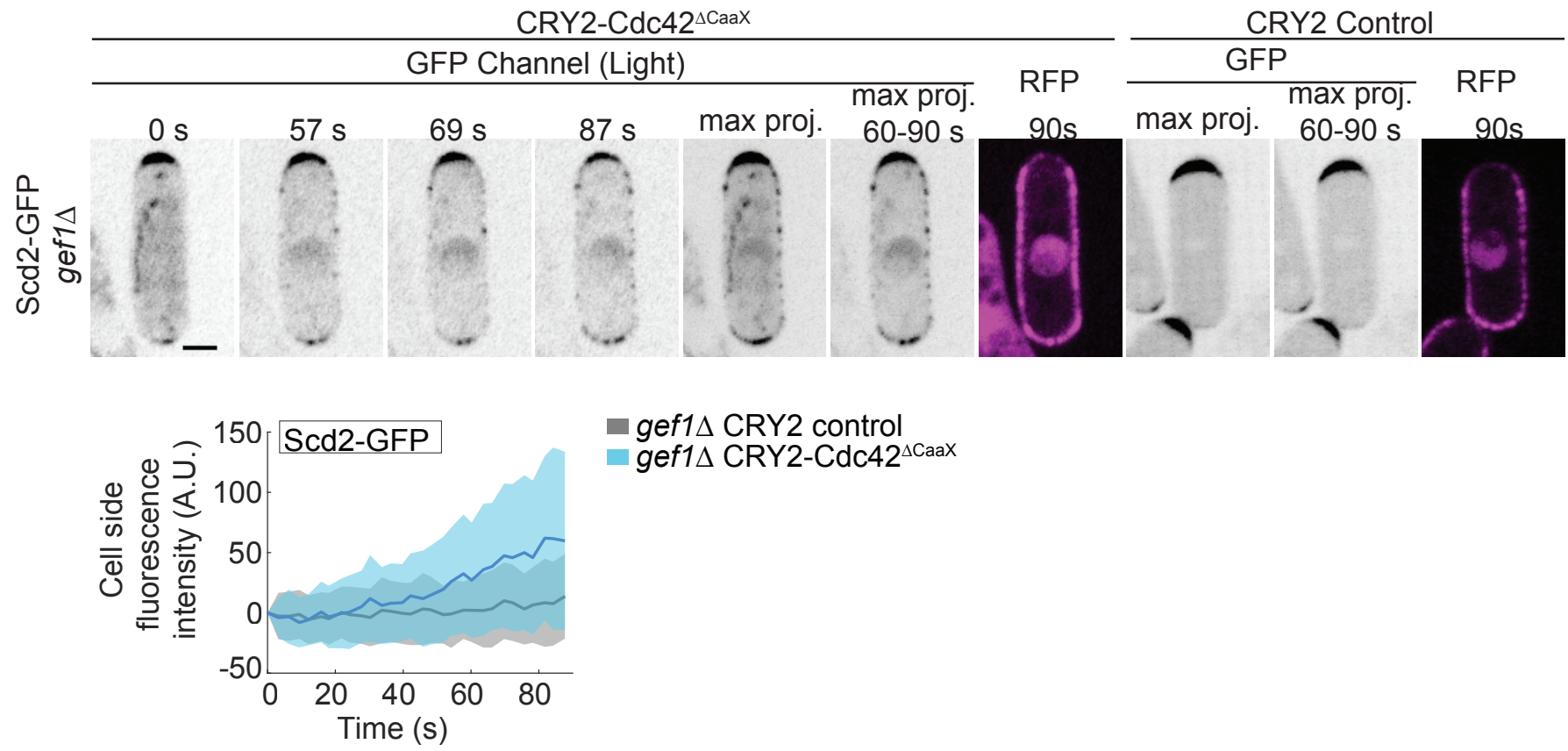

D

gef1 $\triangle$ CRY2 control

gef1 $\triangle$ CRY2-Cdc42 ${ }^{\Delta \mathrm{Caa}}$ 
Figure 3 bioRxiv preprint doi: https://doi.org/10.1101/2020.06.02.130336; this version posted June 2, 2020. The copyright holder for this preprint (which was not certified by peer review) is the author/funder, who has granted bioRxiv a license to display the preprint in perpetuity. It is made available under aCC-BY-NC 4.0 International license.

A

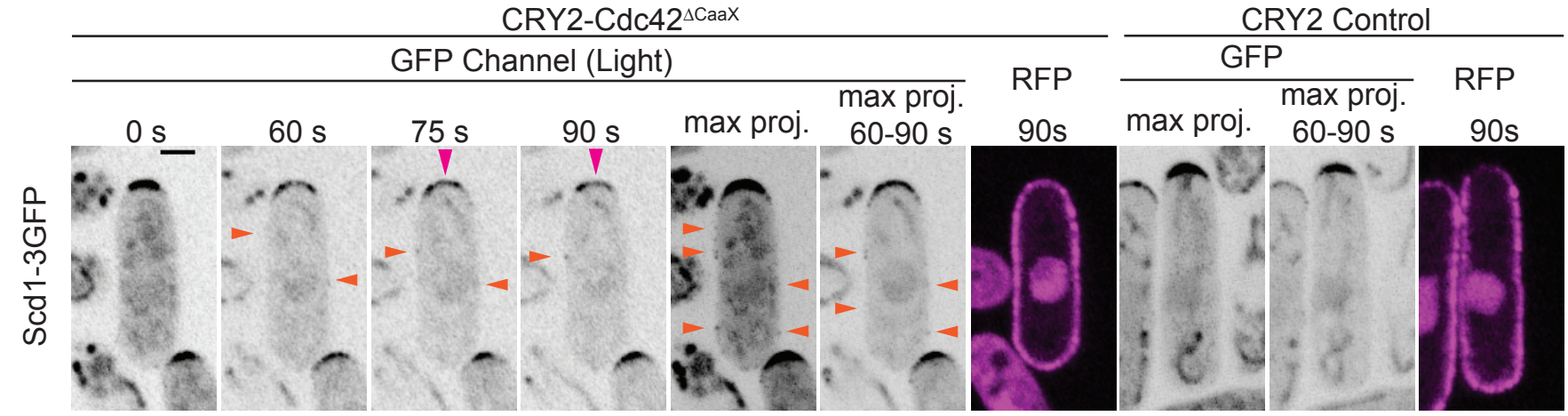

B



- CRY2 control CRY2-Cdc42 ${ }^{\Delta \mathrm{CaaX}}$

D

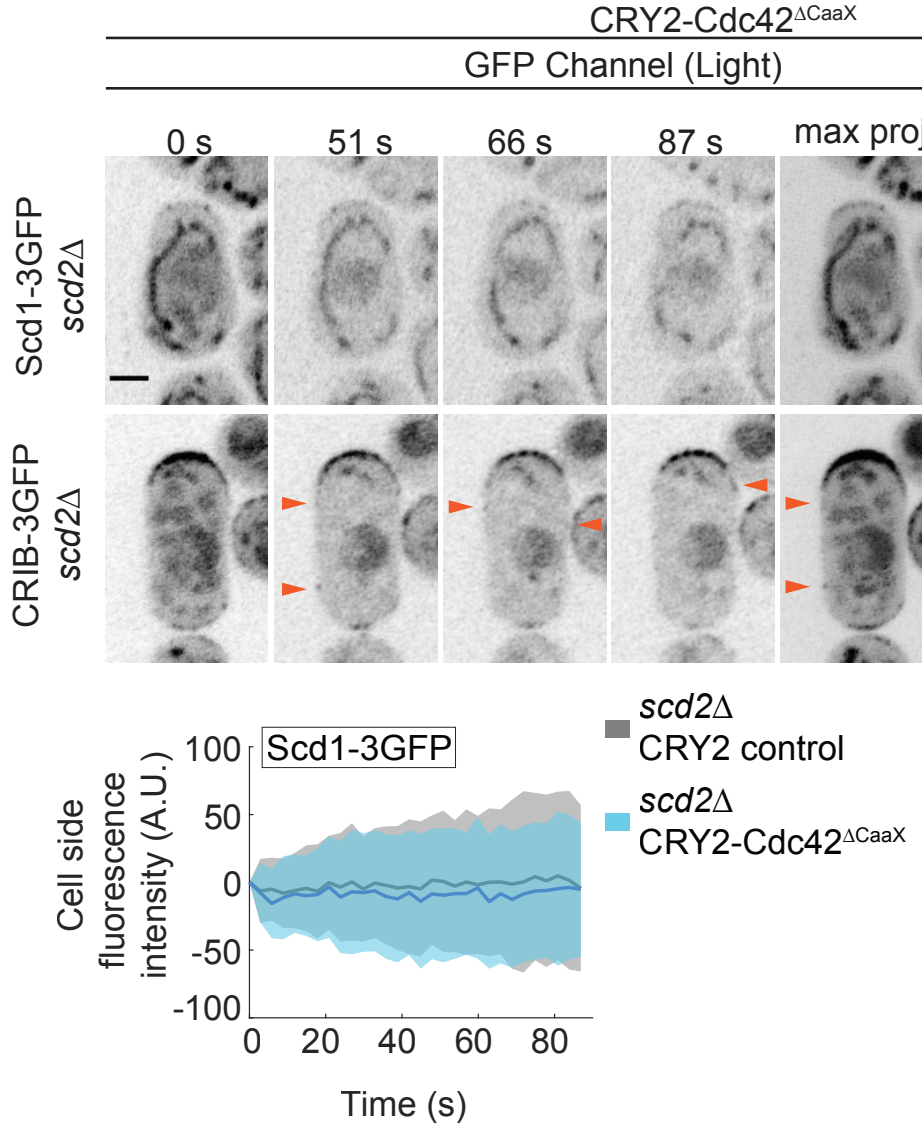

G

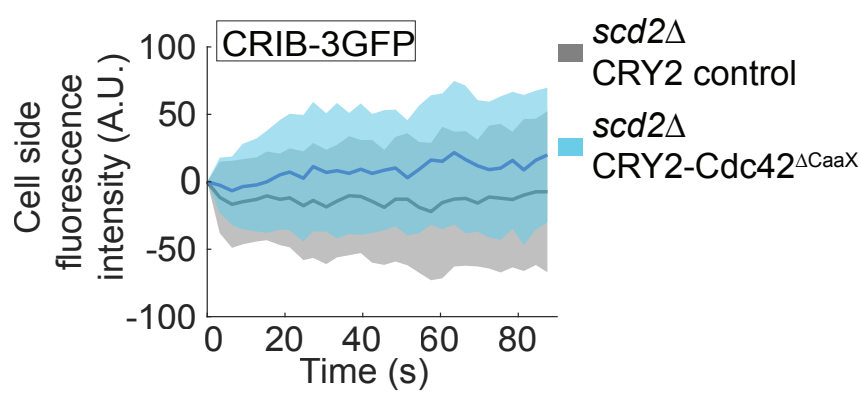

C

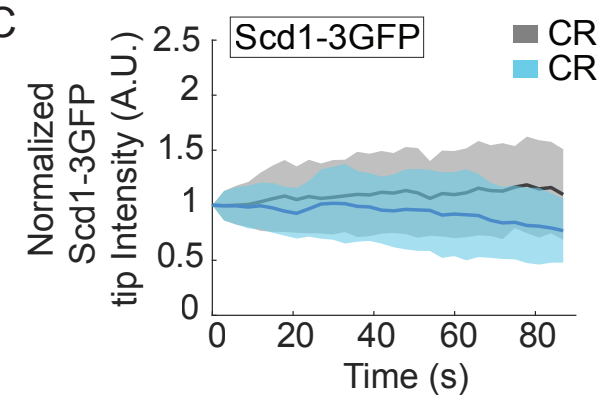

CRY2-Cdc42 ${ }^{\Delta \mathrm{CaaX}}$

CRY2 Control

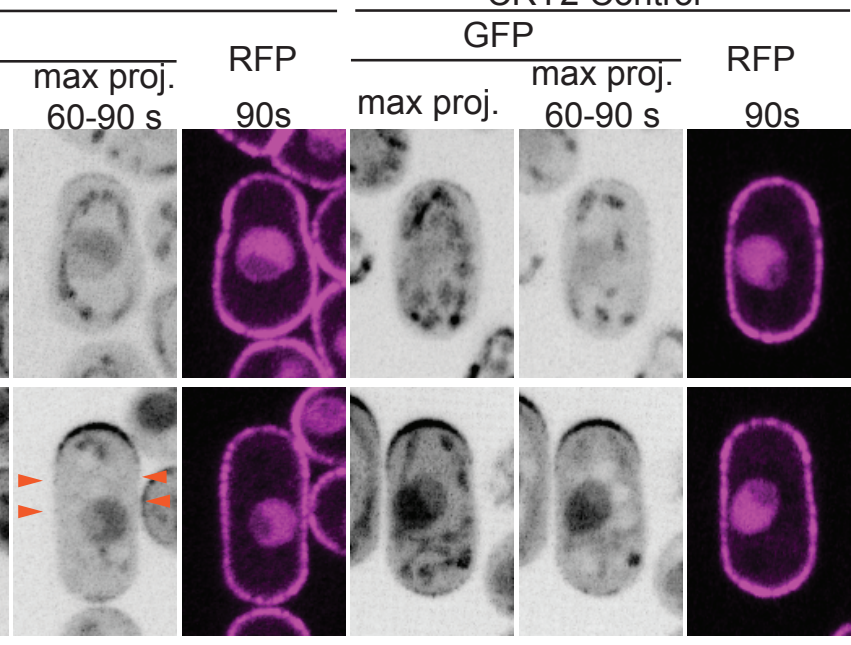

$\mathrm{F}$

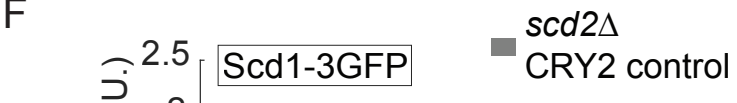

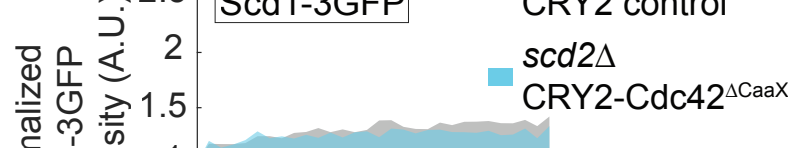
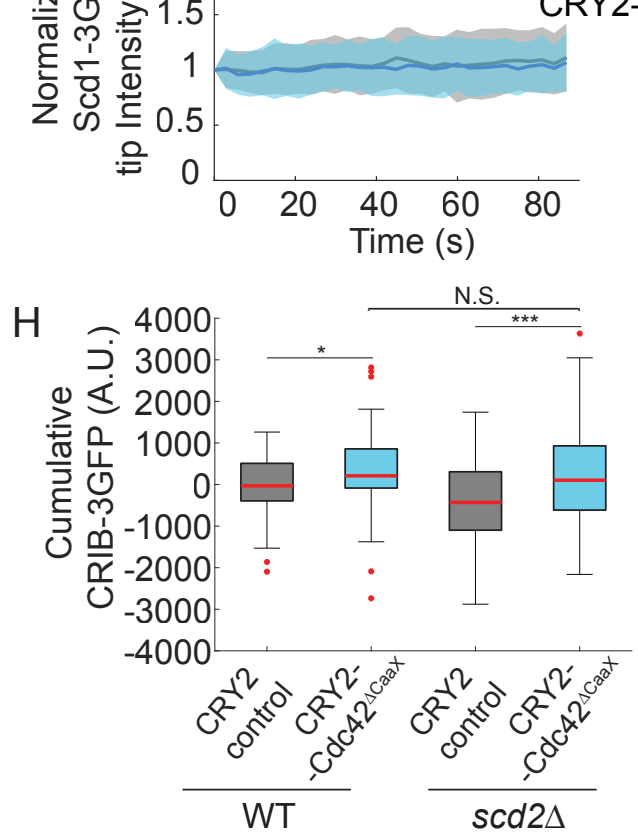
bioRxiv preprint doi: https://doi org/10.1101/2020 06 02 130336; this version posted June 2, 2020. The copyright holder for this preprint Figure( made available under aCC-BY-NC 4.0 International license.

\begin{tabular}{|c|c|c|c|c|c|c|c|c|c|}
\hline & & Cell length & & & Cell width & & & Aspect ratio & \\
\hline Genotype & $\begin{array}{c}\text { CRY2 } \\
\text { control }\end{array}$ & $\begin{array}{l}\text { CRY2- } \\
\text { Cdc42 } \\
\Delta \text { CaaX }\end{array}$ & $\begin{array}{c}\text { CRY2- } \\
\text { Cdc42Q61L, } \\
\Delta \mathrm{CaaX} \\
\end{array}$ & $\begin{array}{c}\text { CRY2 } \\
\text { control } \\
\end{array}$ & $\begin{array}{l}\text { CRY2- } \\
\text { Cdc42 } \\
\Delta \text { CaaX }\end{array}$ & $\begin{array}{c}\text { CRY2- } \\
\text { Cdc42Q61L, } \\
\Delta \text { CaaX }\end{array}$ & $\begin{array}{c}\text { CRY2 } \\
\text { control }\end{array}$ & $\begin{array}{l}\text { CRY2- } \\
\text { Cdc42 } \\
\Delta \text { CaaX }\end{array}$ & \begin{tabular}{|c} 
CRY2- \\
Cdc42Q61L \\
$\Delta$ CaaX \\
\end{tabular} \\
\hline$W T$ & $15.1 \pm 0.09$ & $15 \pm 0.04$ & $11.7 \pm 0.07$ & $3.5 \pm 0.02$ & $3.7 \pm 0.02$ & $5.6 \pm 0.04$ & $4.3 \pm 0.033$ & $4.1 \pm 0.04$ & $2.1 \pm 0.02$ \\
\hline rga $4 \Delta$ & $13.8 \pm 0.1$ & $13.7 \pm 0.04$ & $11.9 \pm 0.09$ & $4 \pm 0.02$ & $4.4 \pm 0.03$ & $5.1 \pm 0.04$ & $3.5 \pm 0.03$ & $3.1 \pm 0.04$ & $2.4 \pm 0.02$ \\
\hline rga6s & $14.2 \pm 0.07$ & $13.9 \pm 0.03$ & $10.2 \pm 0.06$ & $4.1 \pm 0.03$ & $4.2 \pm 0.03$ & $5.7 \pm 0.05$ & $3.5 \pm 0.03$ & $3.4 \pm 0.03$ & $1.8 \pm 0.02$ \\
\hline rga3s & $14.1 \pm 0.08$ & $14.1 \pm 0.03$ & $11.5 \pm 0.07$ & $3.5 \pm 0.03$ & $3.8 \pm 0.03$ & $4.7 \pm 0.05$ & $4.0 \pm 0.03$ & $3.8 \pm 0.03$ & $2.5 \pm 0.02$ \\
\hline rdi1 $\Delta$ & $13.5 \pm 0.09$ & $12.7 \pm 0.02$ & $10.0 \pm 0.05$ & $3.9 \pm 0.03$ & $3.8 \pm 0.02$ & $5.9 \pm 0.02$ & $3.5 \pm 0.03$ & $3.4 \pm 0.02$ & $1.7 \pm 0.02$ \\
\hline gap1A & $13.9 \pm 0.10$ & $13.7 \pm 0.03$ & $9 \pm 0.11$ & $4 \pm 0.02$ & $4 \pm 0.02$ & $7.2 \pm 0.06$ & $3.5 \pm 0.03$ & $3.5 \pm 0.03$ & $1.3 \pm 0.02$ \\
\hline rga4 4 rga $3 \Delta$ & $11 \pm 0.09$ & $11.1 \pm 0.02$ & $11 \pm 0.07$ & $4.4 \pm 0.02$ & $4.6 \pm 0.03$ & $4.8 \pm 0.03$ & $2.7 \pm 0.02$ & $2.4 \pm 0.02$ & $2.3 \pm 0.02$ \\
\hline rga4 4 rga $6 \Delta$ & $11.4 \pm 0.06$ & $10.1 \pm 0.02$ & $9.6 \pm 0.08$ & $5.2 \pm 0.05$ & $5.7 \pm 0.07$ & $6 \pm 0.05$ & $2.2 \pm 0.02$ & $1.8 \pm 0.02$ & $1.6 \pm 0.02$ \\
\hline 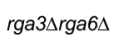 & $13.9 \pm 0.09$ & $13.2 \pm 0.02$ & $10.5 \pm 0.09$ & $4.1 \pm 0.03$ & $4 \pm 0.02$ & $6.3 \pm 0.05$ & $3.4 \pm 0.03$ & $3.3 \pm 0.02$ & $1.7 \pm 0.02$ \\
\hline rdi1 $\Delta r g a 4 \Delta$ & $12.1 \pm 0.07$ & $11.3 \pm 0.02$ & $9.9 \pm 0.06$ & $4.4 \pm 0.02$ & $5.1 \pm 0.03$ & $6.1 \pm 0.06$ & $2.7 \pm 0.02$ & $2.2 \pm 0.02$ & $1.7 \pm 0.02$ \\
\hline rdit $\Delta r g a 6 \Delta$ & $13.1 \pm 0.07$ & $12.9 \pm 0.02$ & $9.4 \pm 0.06$ & $4.2 \pm 0.02$ & $4.3 \pm 0.02$ & $6.3 \pm 0.04$ & $3 \pm 0.02$ & $3 \pm 0.02$ & $1.5 \pm 0.01$ \\
\hline rdi1 $\Delta r g a 3 \Delta$ & $13.8 \pm 0.07$ & $13.2 \pm 0.02$ & $10.6 \pm 0.05$ & $4.3 \pm 0.02$ & $4.2 \pm 0.03$ & $5.4 \pm 0.04$ & $3.2 \pm 0.02$ & $3.2 \pm 0.02$ & $2 \pm 0.02$ \\
\hline gap1 $1 \Delta r g a 4 \Delta$ & $12 \pm 0.11$ & $12.2 \pm 0.02$ & $9.8 \pm 0.11$ & $5.5 \pm 0.04$ & $6.1 \pm 0.05$ & $7.8 \pm 0.07$ & $2.2 \pm 0.02$ & $2.0 \pm 0.02$ & $1.3 \pm 0.01$ \\
\hline gap1 $\Delta r g a 6 \Delta$ & $13.1 \pm 0.07$ & $13 \pm 0.02$ & $9.7 \pm 0.11$ & $4.3 \pm 0.02$ & $4.4 \pm 0.02$ & $8.1 \pm 0.08$ & $3.1 \pm 0.02$ & $3 \pm 0.02$ & $1.1 \pm 0.01$ \\
\hline gap1 $1 \Delta r g a 3 \Delta$ & $13.5 \pm 0.08$ & $12.2 \pm 0.02$ & $9 \pm 0.10$ & $4.5 \pm 0.02$ & $3.9 \pm 0.03$ & $7 \pm 0.05$ & $3.3 \pm 0.02$ & $3.4 \pm 0.02$ & $1.4 \pm 0.01$ \\
\hline
\end{tabular}

B

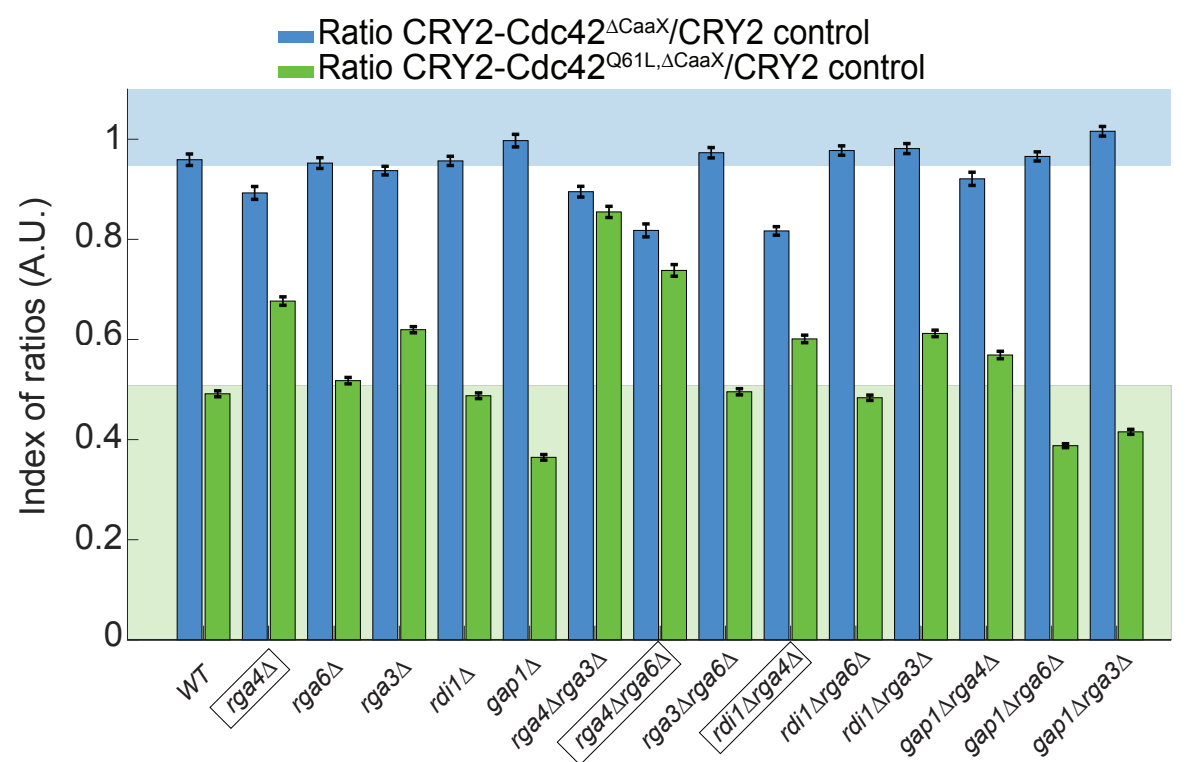

C

Individual cells Average
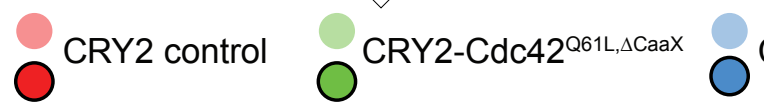

CRY2-Cdc42 4 CaaX
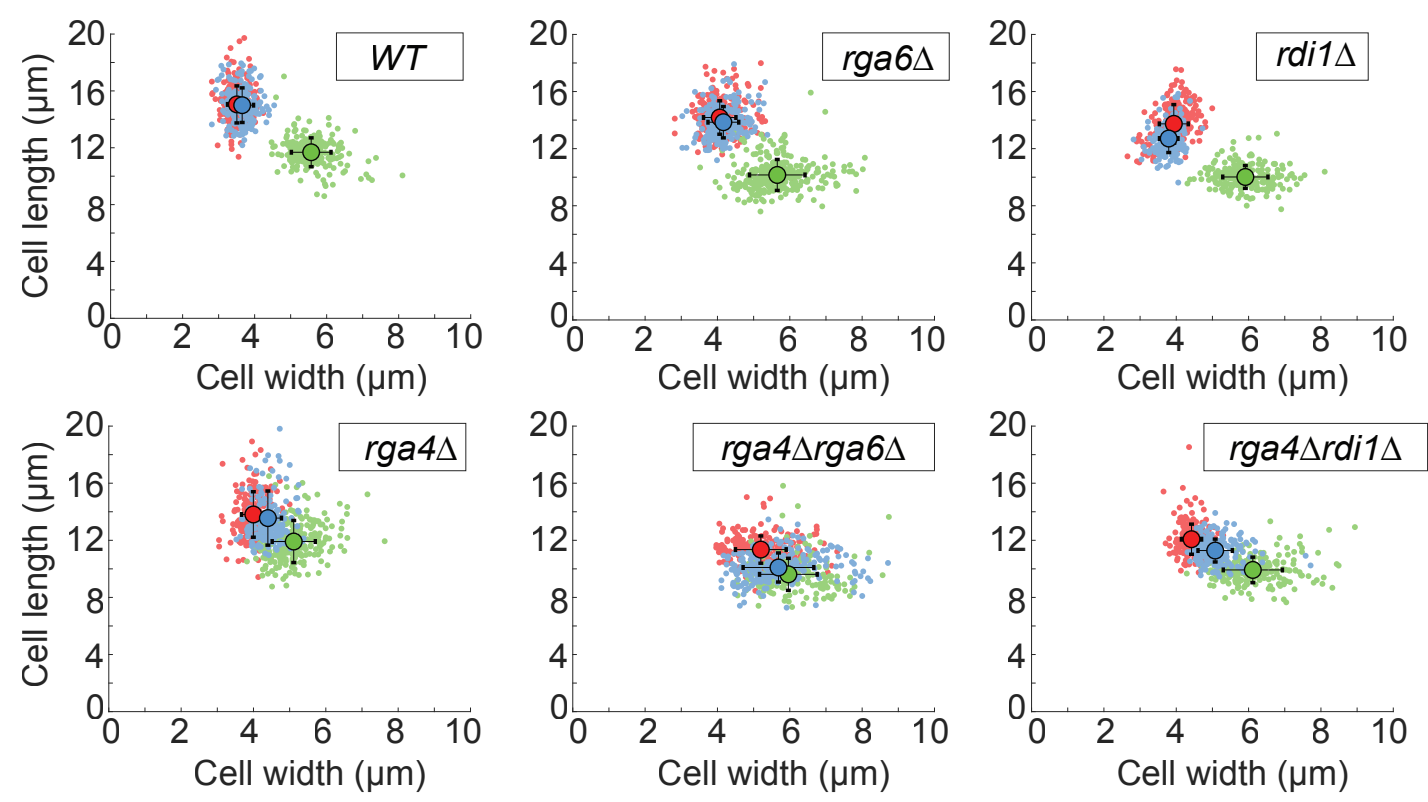
bioRxiv preprint doi: https://doi.org/10.1101/2020.06.02 130336. this version posted June 2 2020. The copyriaht holder for this preprint

Figure Supich was not certified by peer review) is the author/funder, who has granted bioRxiv a license to display the preprint in perpetuity. It is made available under aCC-BY-NC 4.0 International license.
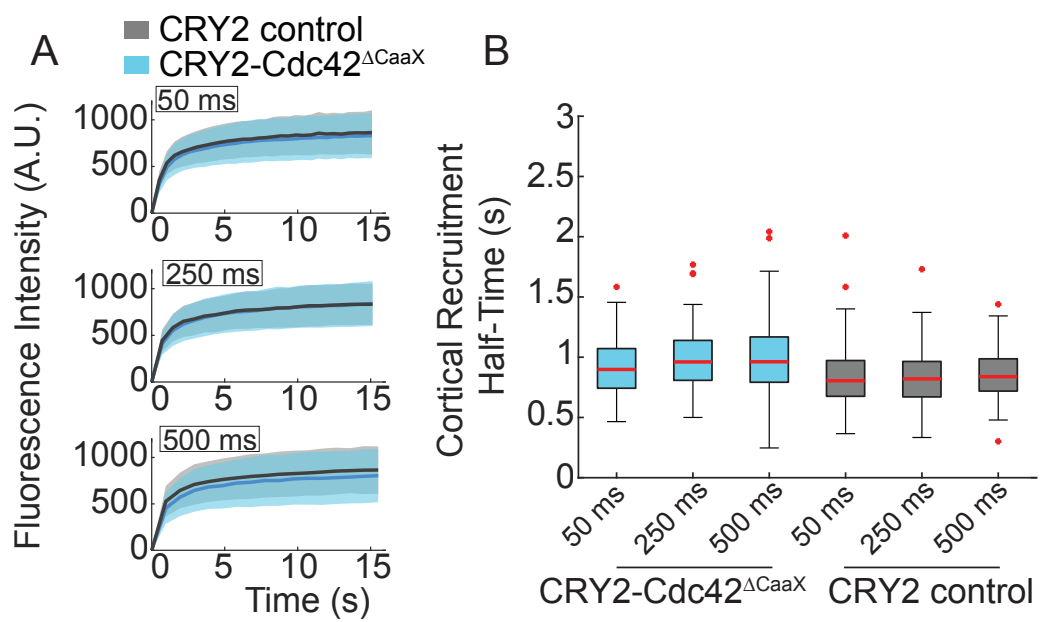

C

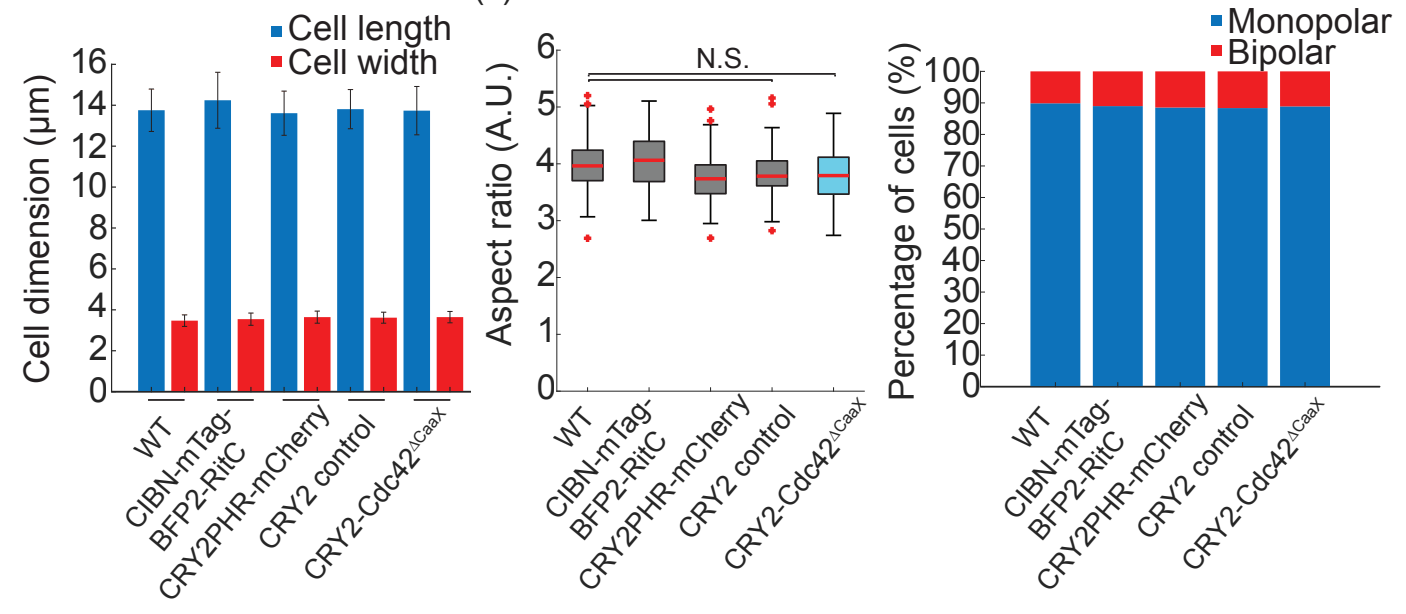

Mix of cells: $\quad$ CRY2-Cdc42 ${ }^{\Delta C a a x}$ (purple) GFP-CIBN-RitC (green)

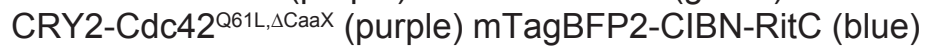

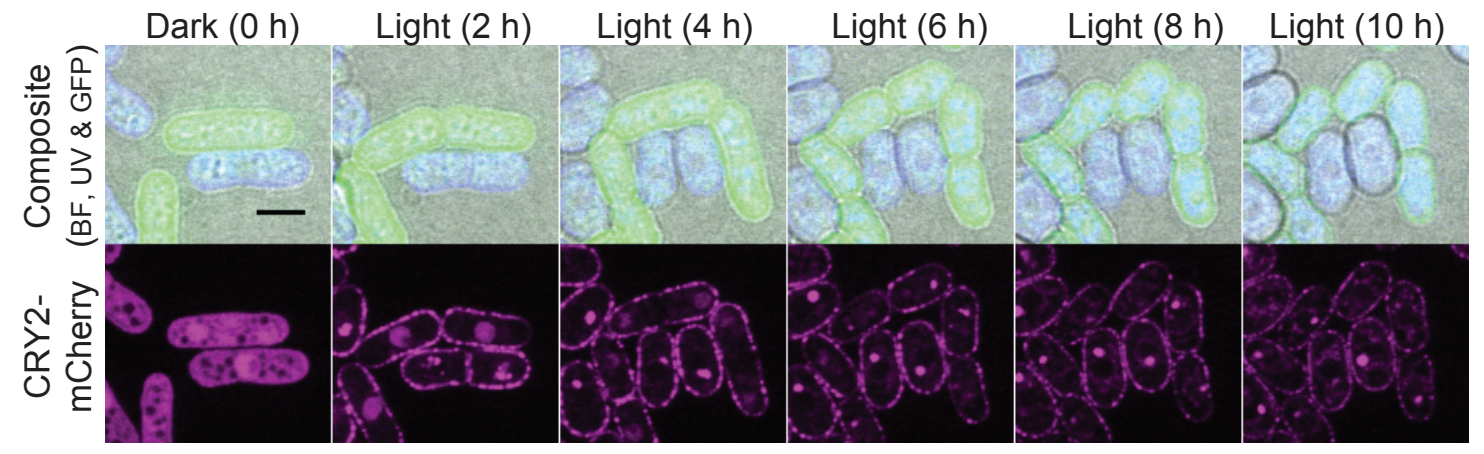


A

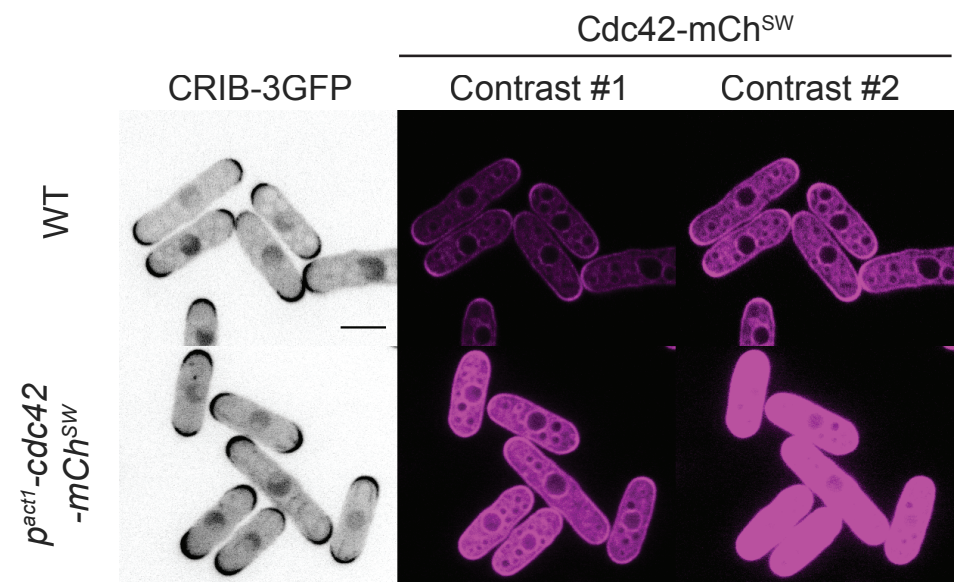

C

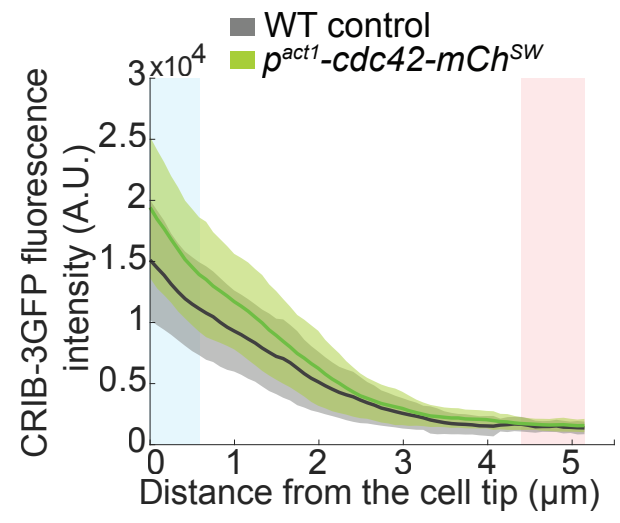

B

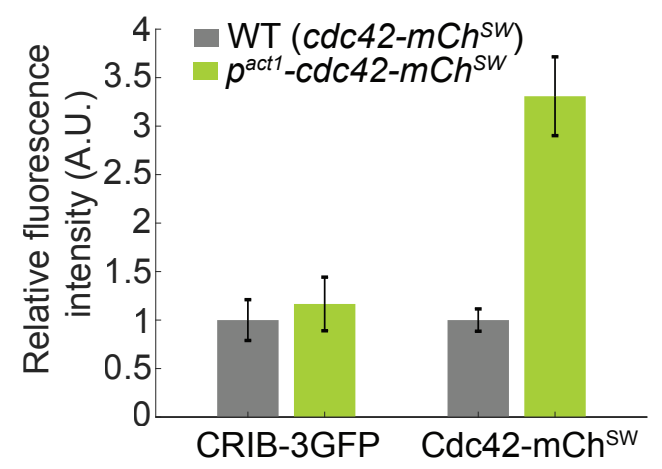

D

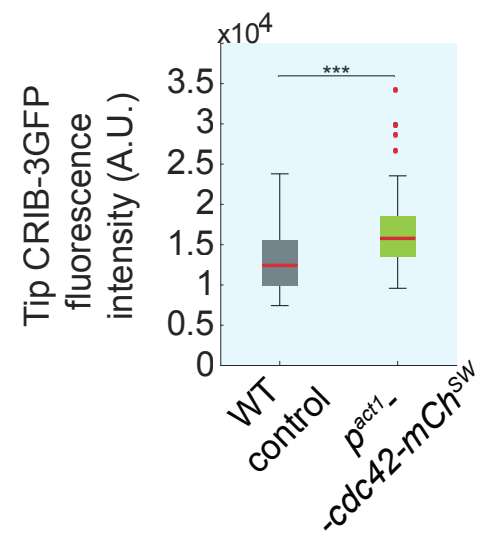


bioRxiv preprint doi: https://doi.org/10.1101/2020.06.02 130336. this version posted June 2 2020. The copyright holder for this preprint

Figure \&Bch was not certified by peer review) is the author/funder, who has granted bioRxiv a license to display the preprint in perpetuity. It is made available under aCC-BY-NC 4.0 International license.

A

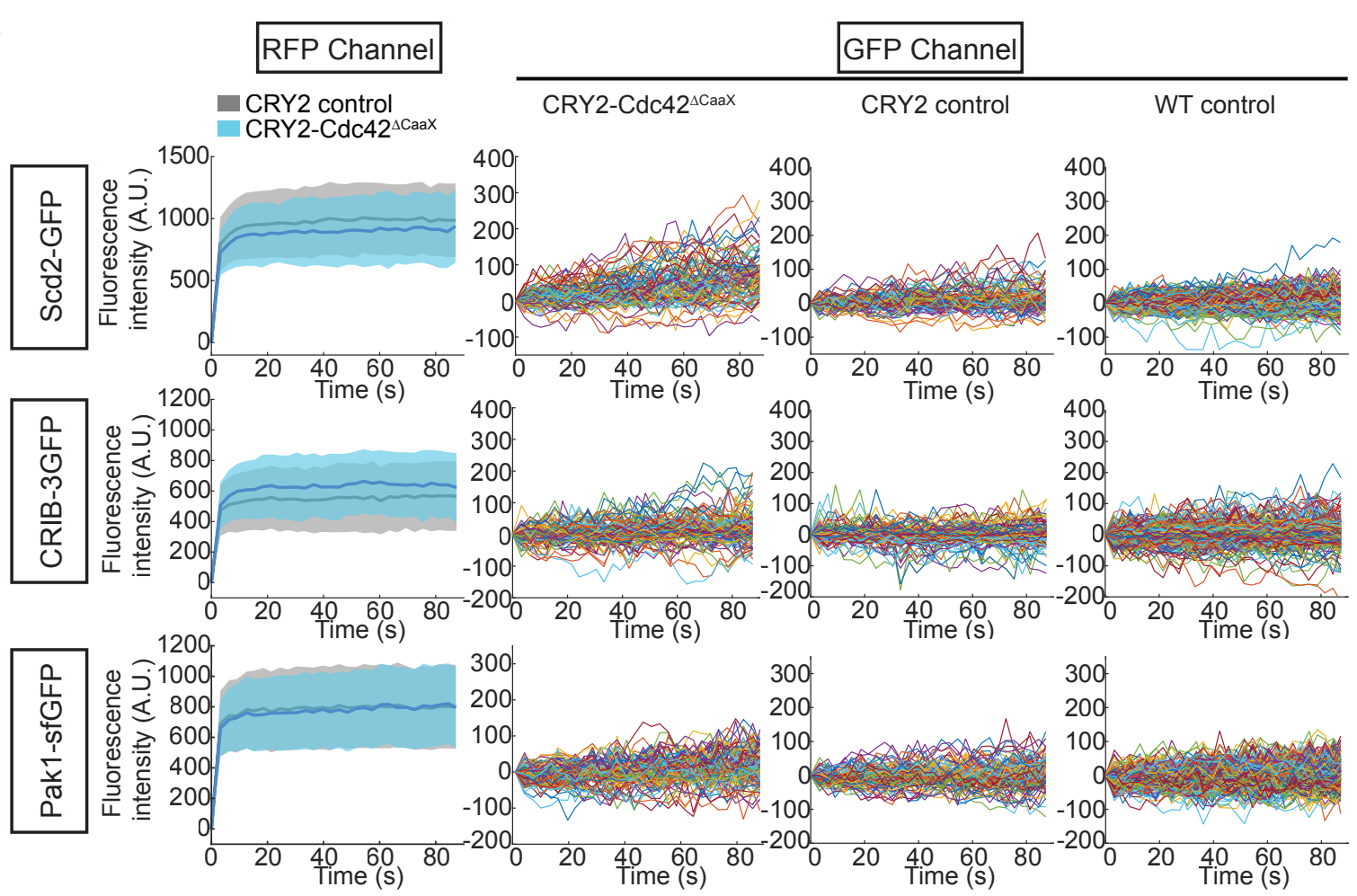

B

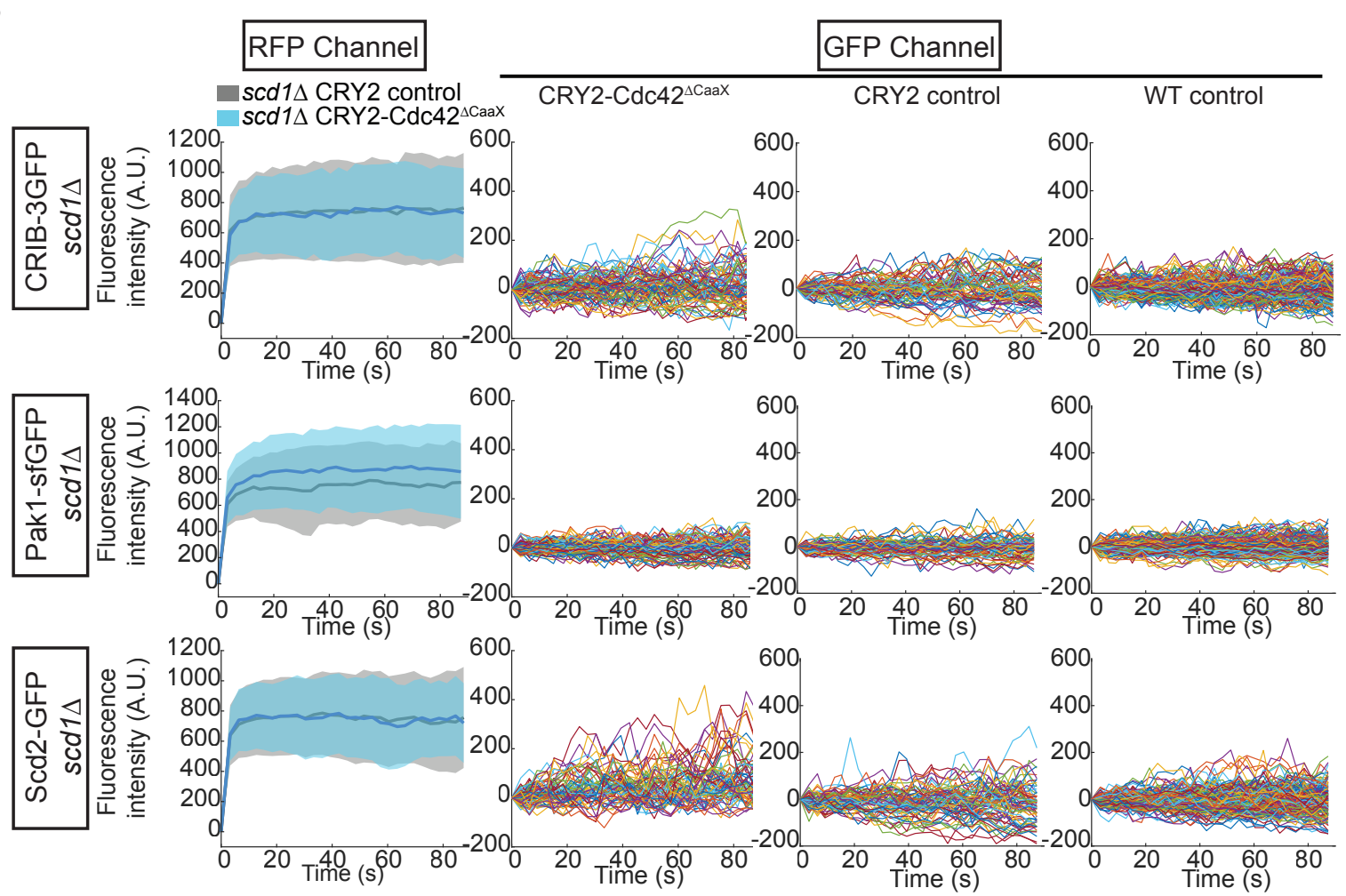

C

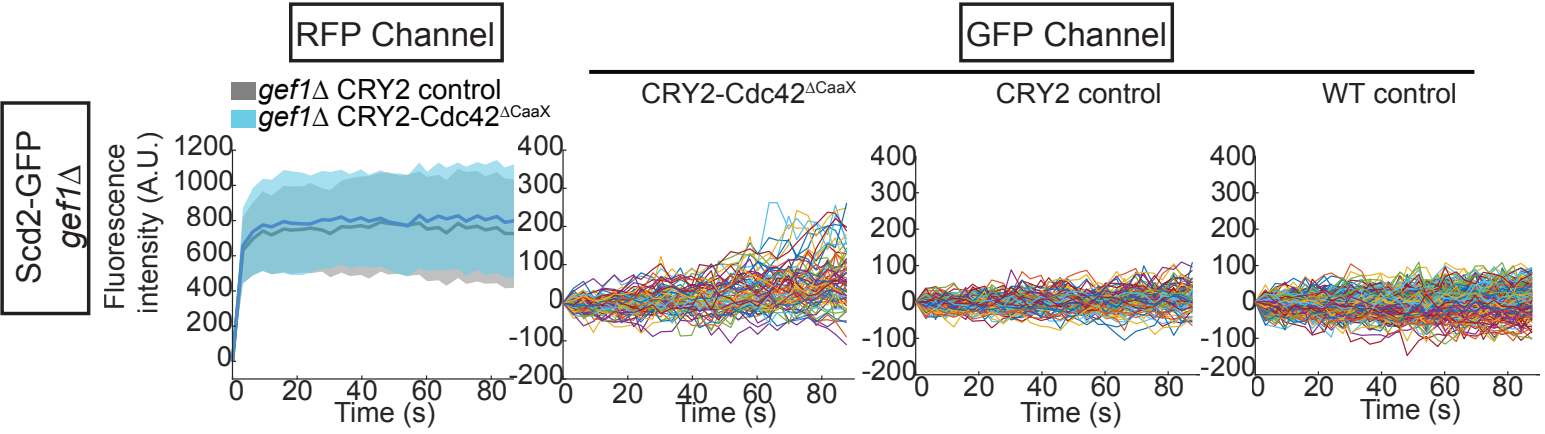


bioRxiv preprint doi: https://doi org/10.1101/2020.06.02 130336: this version posted June 2 2020. The copyriaht holder for this preprint

Figure \$4ich was not certified by peer review) is the author/funder, who has granted bioRxiv a license to display the preprint in perpetuity. It is made available under aCC-BY-NC 4.0 International license.

A

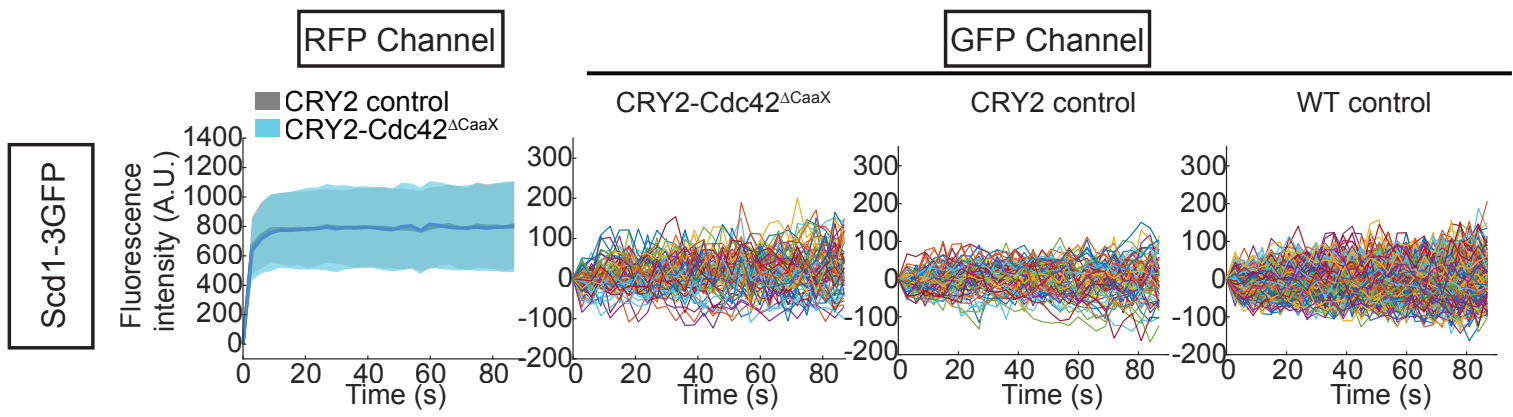

B

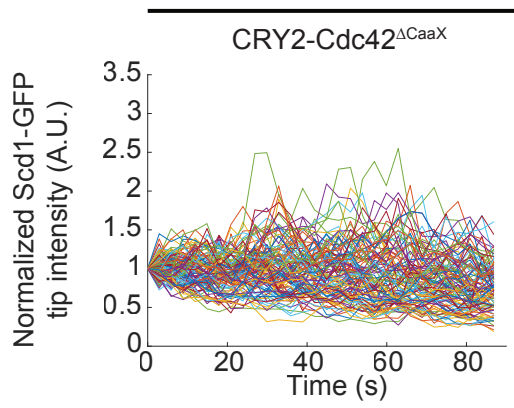

GFP Channel
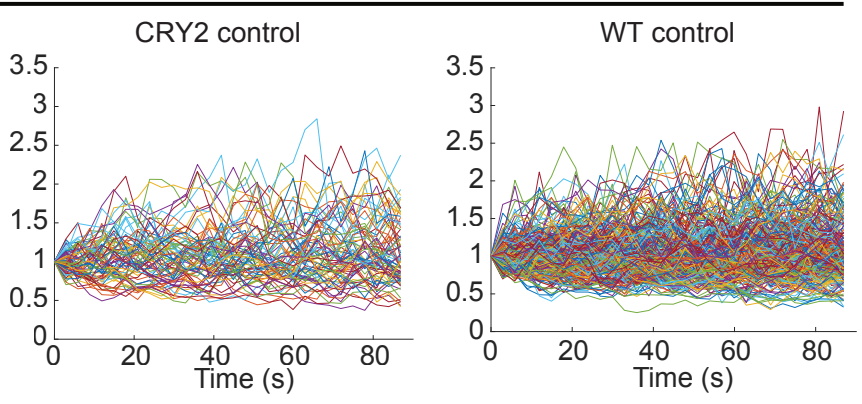

C

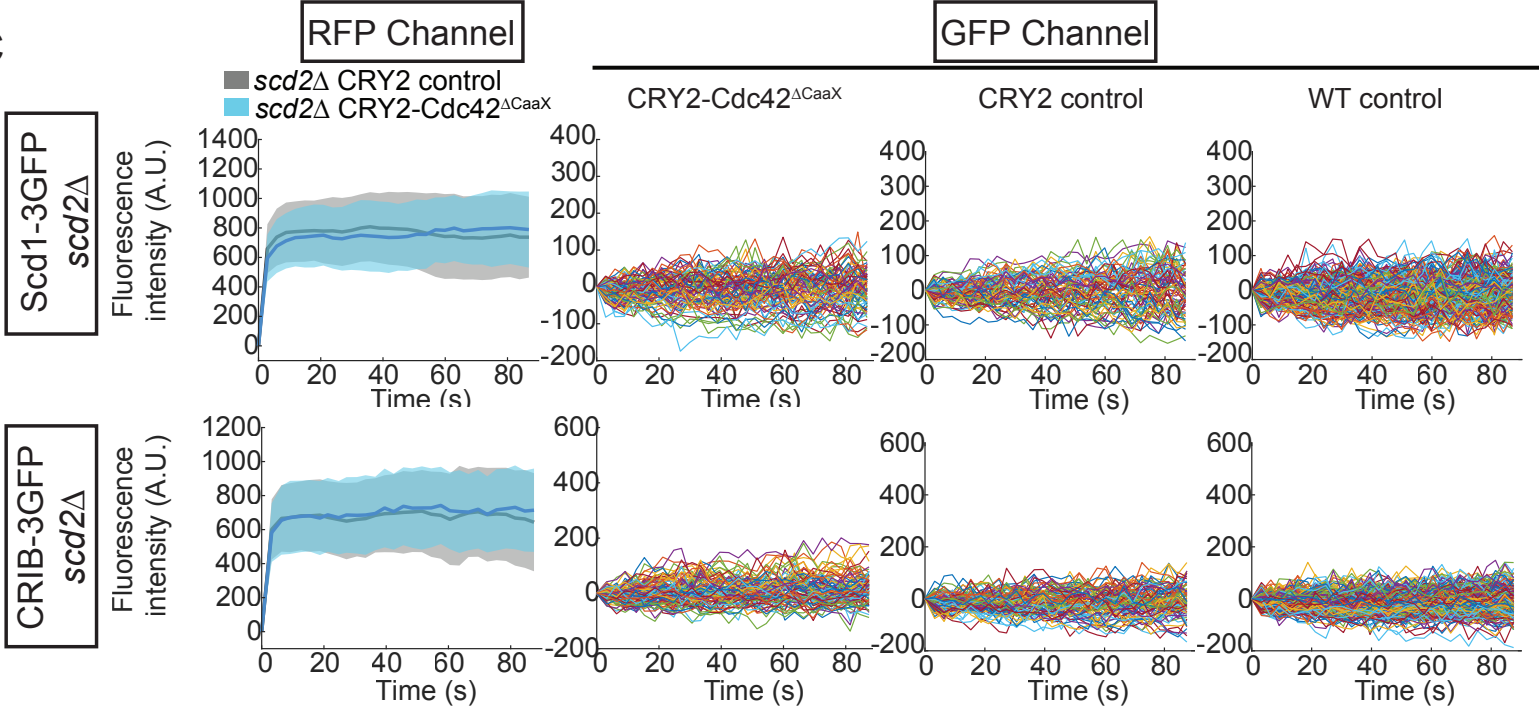

D

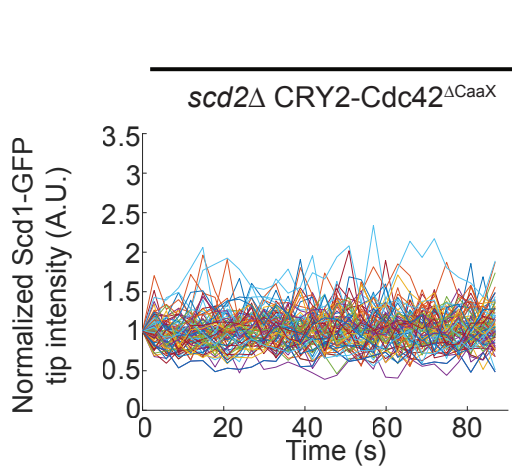

\section{GFP Channel}
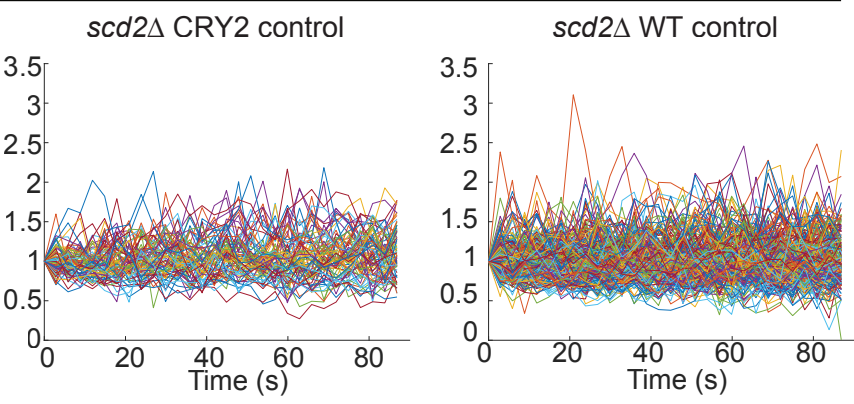
bioRxiv preprint doi: $\mathrm{https}$ //doi.org/10.1101/2020.06.02.130336; this version posted June 2, 2020. The copyright holder for this preprint (which was not certified by peer review) is the author/funder, who has granted bioRxiv a license to display the preprint in perpetuity. It is made available under aCC-BY-NC 4.0 International license.

Table S1

Figure 1 \& Figure S3A

YSM3568 h-leu1-32::Ppak1-CRIB-3xGFP-kanMX+-leu1+ ura4-D18::Ptdh1-CIBN-mTagBFP2-ritc-Scadh1term-Pact1-CRY2PHR-mCherry-ura4 ade6+leu1-32

YSM3730 h-leu1-32::Ppak1-CRIB-3xGFP-kanMX+-leu1+ ura4-D18::Ptdh1-CIBN-mTagBFP2-ritc-Scadh1term-Pact1-CRY2PHR-mCherrycdc42WT $\triangle$ CaaX-ura4 ade6+ leu1-32 ura4-D18

YSM3570 h-leu1-32::Ppak1-CRIB-3XGFP-kanMX+-leu1+ ura4-D18::Ptdh1-CIBN-mTagBFP2-ritc-Scadh1term-ura4+ ade6+leu1-32 ura4-D18

YSM3571 h- pak1::Ppak1-pak1wt-sfGFP-kanMX ura4-294::Ptdh1-CIBN-mTagBFP2-ritc-Scadh1term-Pact1-CRY2PHR-mCherry-ura4+ ade6+ leu1+ura4-294

YSM3731 h- pak1::Ppak1-pak1wt-sfGFP-kanMX ura4-294::Ptdh1-CIBN-mTagBFP2-ritc-Scadh1term-Pact1-CRY2PHR-mCherry-cdc42WTACaaXura4+ ade6+leu1+ ura4-294

YSM3573 h-pak1::Ppak1-pak1wt-sfGFP-kanMX ura4-294::Ptdh1-CIBN-mTagBFP2-ritc-Scadh1term-ura4+ ade6+ leu1+ ura4-294

YSM3574 h- scd2-GFP-kan+ ura4-294::Ptdh1-CIBN-mTagBFP2-ritc-Scadh1term-Pact1-CRY2PHR-mCherry-ura4+ ade6+leu1+ ura4-294

YSM3687 h- scd2-GFP-kan+ ura4-294::Ptdh1-CIBN-mTagBFP2-ritc-Scadh1term-Pact1-CRY2PHR-mCherry-cdc42WTACaaX-ura4+ ade6+leu1+ ura4-294

YSM3576 h-scd2-GFP-kan+ ura4-294::Ptdh1-CIBN-mTagBFP2-ritc-Scadh1term-ura4+ ade6+ leu1+ ura4-294

Figure 2 \& Figure S3B-C

YSM3733

scd1::Nat+ leu1-32::Ppak1-CRIB-3xGFP-KanMX+:leu1+ ura4-D18::Ptdh1-CIBN-mTagBFP2-RitC-ADH1term:Ura4+ ade6+ leu1-32 ura4-D18

YSM3734

Nat+ leu1-32::Ppak1-CRIB-3xGFP-KanMX+:leu1+ ur

YSM3735

mCherry:Ura4+ ade6+ leu1-32 ura4-D18

YSM3613 scd1::natMX+ pak1::Ppak1-pak1wt-sfGFP-kanMX ura4-::Ptdh1-CIBN-mTagBFP2-ritc-Scadh1term-ura4+ ade6+ leu1+ ura4-

YSM3736 scd1::Nat+ pak1::Ppak1-pak1wt-sfGFP-KanMX ura4-::Ptdh1-CIBN-mTagBFP2-Ritc-ADH1term-Pact1-CRY2PHR-mCherrycdc42wt $\triangle$ CaaX:Ura4+ ade6+ leu1+ ura4-

YSM3611 scd1::Nat+ pak1::Ppak1-pak1wt-sfGFP-KanMX ura4-::Ptdh1-CIBN-mTagBFP2-Ritc-ADH1term-Pact1-CRY2PHR-mCherry:Ura4+ ade6+ leu1+ ura4-

YSM3594 scd2-GFP-NatMX scd1::kanMX ura4-::Ptdh1-CIBN-mTagBFP2-RitC-ADH1term:Ura4+ ade6+ leu1+ ura4-

YSM3737 scd2-GFP-NatMX scd1::kanMX ura4-::Ptdh1-CIBN-mTagBFP2-RitC-ADH1term-Pact1-CRY2PHR-mCherry-cdc42WTACaaX:Ura4+ ade6+ leu1+

YSM3592 scd2-GFP-NatMX scd1::kanMX ura4-::Ptdh1-CIBN-mTagBFP2-RitC-ADH1term-Pact1-CRY2PHR-mCherry:Ura4+ ade6+ leu1+ ura4-

YSM3597 gef1::Kan+ scd2-GFP-Nan+ ura4-D18::Ptdh1-CIBN-mTagBFP2-RitC-ADH1term:Ura4+ ade6+ leu1+ ura4-D18

YSM3738 gef1::Kan+ scd2-GFP-Nan+ ura4-D18::Ptdh1-CIBN-mTagBFP2-Ritc-ADH1term-Pact1-CRY2PHR-mCherry-cdc42wtLCaaX:Ura4+ ade6+ leu1+ ura4-D18

YSM3595

gef1::Kan+ scd2-GFP-Nan+ ura4-D18::Ptdh1-CIBN-mTagBFP2-Ritc-ADH1term-Pact1-CRY2PHR-mCherry:Ura4+ ade6+ leu1+ ura4D18

\section{Figure 3 \& Figure $\$ 4$}

YSM3577 h+ scd1-3xGFP-natMX+ ura4-D18::Ptdh1-CIBN-mTagBFP2-ritc-Scadh1term-Pact1-CRY2PHR-mCherry-ura4+ ade6+ leu1+ ura4-D18

YSM3739 $h+$ scd1-3xGFP-natMX+ ura4-D18::Ptdh1-CIBN-mTagBFP2-ritc-Scadh1term-Pact1-CRY2PHR-mCherry-cdc42WTACaaX-ura4+ ade6+ leu1+ ura4-D18

YSM3579 $h+$ scd1-3xGFP-natMX+ ura4-D18::Ptdh1-CIBN-mTagBFP2-ritc-Scadh1term-ura4+ ade6+ leu1+ ura4-D18

YSM3583 scd2::natMX+ leu1-32::Ppak1-CRIB-3xGFP-kanMX+-leu1+ ura4-D18::Ptdh1-CIBN-mTagBFP2-ritc-Scadh1term-Pact1-CRY2PHR$m$ Cherry-ura4+ ade6+ leu1-32 ura4-D18

YSM3740 scd2::natMX+ leu1-32::Ppak1-CRIB-3xGFP-kanMX+-leu1+ ura4-D18::Ptdh1-CIBN-mTagBFP2-ritc-Scadh1term-Pact1-CRY2PHR$m$ Cherry-cdc42WT $\triangle$ CaaX-ura4+ ade6+ leu1-32 ura4-D18

YSM3585 scd2::natMX+ leu1-32::Ppak1-CRIB-3xGFP-kanMX+-leu1+ ura4-D18::Ptdh1-CIBN-mTagBFP2-ritc-Scadh1term-ura4+ ade6+ leu1-32 ura4-D18

YSM3586 scd2::natMX pak1::Ppak1-pak1wt-sfGFP-kanMX ura4-294::Ptdh1-CIBN-mTagBFP2-ritc-Scadh1term-Pact1-CRY2PHR-mCherry-ura4+ ade6+leu1+ ura4-294

YSM3741 scd2::natMX pak1::Ppak1-pak1wt-sfGFP-kanMX ura4-294::Ptdh1-CIBN-mTagBFP2-ritc-Scadh1term-Pact1-CRY2PHR-mCherrycdc42WT $\Delta$ CaaX-ura4+ ade6+ leu1+ ura4-294

YSM3588 scd2::natMX pak1::Ppak1-pak1wt-sfGFP-kanMX ura4-294::Ptdh1-CIBN-mTagBFP2-ritc-Scadh1term-ura4+ ade6+ leu1+ ura4-294 YSM3589 scd1-3xGFP-natMX scd2::natMX ura4-D18::Ptdh1-CIBN-mTagBFP2-ritc-Scadh1term-Pact1-CRY2PHR-mCherry-ura4+ ade6+ leu1+ ura4-D18

YSM3742 scd1-3xGFP-natMX scd2::natMX ura4-D18::Ptdh1-CIBN-mTagBFP2-ritc-Scadh1term-Pact1-CRY2PHR-mCherry-cdc42WT $\triangle$ CaaX-ura4+ ade6+leu1+ ura4-D18

YSM3591 scd1-3xGFP-natMX scd2::natMX ura4-D18::Ptdh1-CIBN-mTagBFP2-ritc-Scadh1term-ura4+ ade6+ leu1+ ura4-D18

Figure 4

YSM3565 h- Ura4-294::Ptdh1-CIBN-mTagBFP2-Ritc-ADH1term-Pact1-CRY2PHR-mCherry:Ura4+ ade6+ leu1+ ura4-294

YSM3566 h- Ura4-294::Ptdh1-CIBN-mTagBFP2-Ritc-ADH1term-Pact1-CRY2PHR-mCherry-cdc42Q61LACaaX:Ura4+ ade6+ leu1+

YSM3743 h- Ura4-294::Ptdh1-CIBN-mTagBFP2-Ritc-ADH1term-Pact1-CRY2PHR-mCherry-cdc42wt CaaX:Ura4+ ade6+ leu1+

YSM3744 h- rga4::Nat ura4-294::Ptdh1-CIBN-mTagBFP2-Ritc-ADH1term-Pact1-CRY2PHR-mCherry:Ura4+ ade6+ leu1+ ura4-294

YSM3745 h- rga4::Nat ura4-294::Ptdh1-CIBN-mTagBFP2-Ritc-ADH1term-Pact1-CRY2PHR-mCherry-cdc42Q61LACaaX:Ura4+ ade6+ leu1+ ura4-294

YSM3746 h- rga4::Nat ura4-294::Ptdh1-CIBN-mTagBFP2-Ritc-ADH1term-Pact1-CRY2PHR-mCherry-cdc42wt $\triangle$ CaaX:Ura4+ ade6+ leu1+ ura4294

YSM3747 h- rga6::kan+ ura4-294::Ptdh1-CIBN-mTagBFP2-Ritc-ADH1term-Pact1-CRY2PHR-mCherry:Ura4+ ade6+ leu1+ ura4-294

YSM3748 h- rga6::kan+ ura4-294::Ptdh1-CIBN-mTagBFP2-Ritc-ADH1term-Pact1-CRY2PHR-mCherry-cdc42Q61LACaaX:Ura4+ ade6+ leu1+ ura4-294

YSM3749 h- rga6::kan+ ura4-294::Ptdh1-CIBN-mTagBFP2-Ritc-ADH1term-Pact1-CRY2PHR-mCherry-cdc42wtACaaX:Ura4+ ade6+ leu1+ ura4294 
bioRxiv preprint doi: $\mathrm{https}$ //doi.org/10.1101/2020.06.02.130336; this version posted June 2, 2020. The copyright holder for this preprint (which was not certified by peer review) is the author/funder, who has granted bioRxiv a license to display the preprint in perpetuity. It is made available under aCC-BY-NC 4.0 International license.

YSM3750 h- rga3::Kan+ ura4-294::Ptdh1-CIBN-mTagBFP2-Ritc-ADH1term-Pact1-CRY2PHR-mCherry:Ura4+ ade6+ leu1+ ura4-294

YSM3751 $h$ - rga3:: Kar

YSM3752 h- rga3::Kan+ ura4-294::Ptdh1-CIBN-mTagBFP2-Ritc-ADH1term-Pact1-CRY2PHR-mCherry-cdc42wt_CaaX:Ura4+ ade6+ leu1+ ura4294

YSM3753 h- rdi1::kan+ ura4-294::Ptdh1-CIBN-mTagBFP2-RitC-ADH1term-Pact1-CRY2PHR-mCherry-Ura4+ ade6+ leu1+ ura4-294

YSM3754 h-rdi1::kan+ ura4-294::Ptdh1-CIBN-mTagBFP2-RitC-ADH1term-Pact1-CRY2PHR-mCherry-Cdc42Q61LCaaX-Ura4+ ade6+ leu1+ ura4-294

YSM3755 h- rdi1::kan+ ura4-294::Ptdh1-CIBN-mTagBFP2-RitC-ADH1term-Pact1-CRY2PHR-mCherry-Cdc42WTCaaX-Ura4+ ade6+ leu1+ ura4294

YSM3756 h- $h$ - gap1::Nat+ ura4-294::Ptdh1-CIBN-mTagBFP2-Ritc-ADH1term-Pact1-CRY2PHR-mCherry:Ura4+ ade6+ leu1+ ura4-294

YSM3757 $h$ - $h$ - gap1::Nat+ ura4-294::Ptdh1-CIBN-mTagBFP2-Ritc-ADH1term-Pact1-CRY2PHR-mCherry-cdc42Q61LACaaX:Ura4+ ade6+ leu1+ ura4-294

YSM3758 $h$ - $h$ - gap1::Nat+ ura4-294::Ptdh1-CIBN-mTagBFP2-Ritc-ADH1term-Pact1-CRY2PHR-mCherry-cdc42wt $\Delta$ CaaX:Ura4+ ade6+ leu1+ ura4-294

YSM3759 rga4::Kan+ rga3::Kan+ ura4-::Ptdh1-CIBN-mTagBFP2-Ritc-ADH1term-Pact1-CRY2PHR-mCherry:Ura4+ ade6+ leu1+ ura4-

YSM3760 rga4::Kan+ rga3::Kan+ ura4-::Ptdh1-CIBN-mTagBFP2-Ritc-ADH1term-Pact1-CRY2PHR-mCherry-cdc42Q61LACaaX:Ura4+ ade6+ leu1+ ura4-

YSM3761 rga4::Kan+ rga3::Kan+ ura4-::Ptdh1-CIBN-mTagBFP2-Ritc-ADH1term-Pact1-CRY2PHR-mCherry-cdc42wt $\triangle$ CaaX:Ura4+ ade6+ leu1+ ura4-

YSM3762 h- rga4::kan rga6::kan Ura4-::Ptdh1-CIBN-mTagBFP2-Ritc-ADH1term-Pact1-CRY2PHR-mCherry:Ura4+ ade6+ leu1+ ura4-

YSM3763 h- rga4::kan rga6::kan Ura4-::Ptdh1-CIBN-mTagBFP2-Ritc-ADH1term-Pact1-CRY2PHR-mCherry-cdc42Q61LACaaX:Ura4+ ade6+ leu1+ ura4-

YSM3764 h- rga4::kan rga6::kan Ura4-::Ptdh1-CIBN-mTagBFP2-Ritc-ADH1term-Pact1-CRY2PHR-mCherry-cdc42wt $\Delta$ CaaX:Ura4+ ade6+ leu1+

YSM3765 rga6::Kan+ rga3::Kan+ ura4-::Ptdh1-CIBN-mTagBFP2-Ritc-ADH1term-Pact1-CRY2PHR-mCherry:Ura4+ ade6+ leu1+ ura4-

YSM3766 rga6::Kan+ rga3::Kan+ ura4-::Ptdh1-CIBN-mTagBFP2-Ritc-ADH1term-Pact1-CRY2PHR-mCherry-cdc42Q61LACaaX:Ura4+ ade6+ leu1+ ura4YSM3767 rga6::Kan+ rga3::Kan+ ura4-::Ptdh1-CIBN-mTagBFP2-Ritc-ADH1term-Pact1-CRY2PHR-mCherry-cdc42wt $\begin{aligned} & \text { uraa- } \\ & \text { Y }\end{aligned}$

YSM3768 rdi1::kan rga4::kan Ura4-::Ptdh1-CIBN-mTagBFP2-Ritc-ADH1term-Pact1-CRY2PHR-mCherry:Ura4+ ade6+ leu1+ ura4-

YSM3769 rdi1::kan rga4::kan Ura4-::Ptdh1-CIBN-mTagBFP2-Ritc-ADH1term-Pact1-CRY2PHR-mCherry-cdc42Q61LACaaX:Ura4+ ade6+ leu1+ ura4-

YSM3770 rdi1::kan rga4::kan Ura4-::Ptdh1-CIBN-mTagBFP2-Ritc-ADH1term-Pact1-CRY2PHR-mCherry-cdc42wtACaaX:Ura4+ ade6+ leu1+ ura4-

YSM3771 rdi1::kan+ rga6::kan+::Ptdh1-CIBN-mTagBFP2-Ritc-ADH1term-Pact1-CRY2PHR-mCherry:Ura4+ ade6+ leu1+ ura4-

YSM3772 rdi1::kan+ rga6::kan+::Ptdh1-CIBN-mTagBFP2-Ritc-ADH1term-Pact1-CRY2PHR-mCherry-cdc42Q61LACaaX:Ura4+ ade6+ leu1+ ura4-

YSM3773 rdi1::kan+ rga6::kan+::Ptdh1-CIBN-mTagBFP2-Ritc-ADH1term-Pact1-CRY2PHR-mCherry-cdc42wt $\Delta$ CaaX:Ura4+ ade6+ leu1+ ura4-

YSM3774 rdi1::kan+ rga3::kan+ Ura4-::Ptdh1-CIBN-mTagBFP2-Ritc-ADH1term-Pact1-CRY2PHR-mCherry:Ura4+ ade6+ leu1+ ura4-

YSM3775 rdi1::kan+ rga3::kan+ Ura4-::Ptdh1-CIBN-mTagBFP2-Ritc-ADH1term-Pact1-CRY2PHR-mCherry-cdc42Q61LACaaX:Ura4+ ade6+ leu1+ ura4-

YSM3776 rdi1::kan+ rga3::kan+ Ura4-::Ptdh1-CIBN-mTagBFP2-Ritc-ADH1term-Pact1-CRY2PHR-mCherry-cdc42wt $\Delta$ CaaX:Ura4+ ade6+ leu1+ ura4-

YSM3777 h- rga4::Nat gap1::Hph+ ura4-294::Ptdh1-CIBN-mTagBFP2-RitC-ADH1term-1-CIBN-mTagBFP2-Ritc-ADH1term-Pact1-CRY2PHRmCherry:Ura4+ ade6+ leu1+ ura4-294

YSM3778 h- rga4::Nat gap1::Hph+ ura4-294::Ptdh1-CIBN-mTagBFP2-RitC-ADH1term-1-CIBN-mTagBFP2-Ritc-ADH1term-Pact1-CRY2PHRmCherry-cdc42Q61L $\triangle$ CaaX:Ura4+ ade6+ leu1+ ura4-294

YSM3779 h- rga4::Nat gap1::Hph+ ura4-294::Ptdh1-CIBN-mTagBFP2-RitC-ADH1term-1-CIBN-mTagBFP2-Ritc-ADH1term-Pact1-CRY2PHRmCherry-cdc42wt $\triangle$ CaaX:Ura4+ ade6+ leu1+ ura4-294

YSM3780 h- rga6::kan+ gap1::hph+ ura4-294::Ptdh1-CIBN-mTagBFP2-RitC-ADH1term-1-CIBN-mTagBFP2-Ritc-ADH1term-Pact1-CRY2PHRmCherry:Ura4+ ade6+ leu1+ ura4-294

YSM3781 h- rga6::kan+ gap1::hph+ ura4-294::Ptdh1-CIBN-mTagBFP2-RitC-ADH1term-1-CIBN-mTagBFP2-Ritc-ADH1term-Pact1-CRY2PHRmCherry-cdc42Q61L $\triangle$ CaaX:Ura4+ ade6+ leu1+ ura4-294

YSM3782 h- rga6::kan+ gap1::hph+ ura4-294::Ptdh1-CIBN-mTagBFP2-RitC-ADH1term-1-CIBN-mTagBFP2-Ritc-ADH1term-Pact1-CRY2PHR$m$ Cherry-cdc42wt $\triangle$ CaaX:Ura4+ ade6+ leu1+ ura4-294

YSM3783 h- rga3::Kan+ gap1::hph+ ura4-294::Ptdh1-CIBN-mTagBFP2-RitC-ADH1term-1-CIBN-mTagBFP2-Ritc-ADH1term-Pact1-CRY2PHRmCherry:Ura4+ ade6+ leu1+ ura4-294

YSM3784 h- rga3::Kan+ gap1::hph+ ura4-294::Ptdh1-CIBN-mTagBFP2-RitC-ADH1term-1-CIBN-mTagBFP2-Ritc-ADH1term-Pact1-CRY2PHRmCherry-cdc42Q61L $\triangle$ CaaX:Ura4+ ade6+ leu1+ ura4-294

YSM3785 h- rga3::Kan+ gap1::hph+ ura4-294::Ptdh1-CIBN-mTagBFP2-RitC-ADH1term-1-CIBN-mTagBFP2-Ritc-ADH1term-Pact1-CRY2PHR$m$ Cherry-cdc42wt $\Delta$ CaaX:Ura4+ ade6+ leu1+ ura4-294

YSM3563 h- ura4-294::Ptdh1-CIBN-mTagBFP2-ritc-Scadh1term-ura4+

YSM3564 h- ura4-294::Pact1-CRY2PHR-mCherry-ura4+

YSM3565 h-ura4-294::Ptdh1-CIBN-mTagBFP2-ritc-Scadh1term-Pact1-CRY2PHR-mCherry-ura4

YSM3566 h-ura4-294::Ptdh1-CIBN-mTagBFP2-ritc-Scadh1term-Pact1-CRY2PHR-mCherry-cdc42Q61LACaaX-ura4

YSM3567 h- ura4-294::Ptdh1-CIBN-GFP-RitC-ADH1term-Pact1-CRY2-mcherry-Cdc42WTACaaX-ura4+ ade6+ leu1+ ura4-294

YSM1372 h-WT (972) ade6+ leu1+ ura4+ his7+

YSM3732 cdc42::hphMX ura4-294-Pact1-cdc42-mCherry-SW-ura4 + leu1-32-Pshk1-ScGIC2 CRIB-3xGFP-leu1+-ura4+ 